\title{
VENT'ANNI DI ELEZIONE DIRETTA DEL SINDACO A BOLOGNA
}

di Marta Regalia e Marco Valbruzzi 


\section{Twenty years of direct mayoral election in Bologna}

Abstract - The article retraces the last 20 years of direct mayoral election in Bologna. It shows how the changes in institutional and electoral settings influenced both the party system and voters' electoral responses. By analysing the electoral history of the city, we also show that personalization raised, electoral competition became more (bi)polarized, electoral turnout gradually decreased, voting results became less and less predictable attenuating Bologna past exceptionalism. This article fills a gap in a literature that has not yet offered a longitudinal study of electoral changes in Bologna since the 1990s. More precisely, the article gives an analysis of both the political supply and the electoral flows to understand how party system has changed and if and how voters have become more mobile.

Keywords: Bologna, elections, Mayor, electoral flows, personalization 


\section{Bologna "normalizzata"}

Da un punto di vista politico ed elettorale, Bologna non è una città come le altre. Per molti anni è stata considerata come la capitale della "subcultura politica rossa", e cioè il cuore di quella tradizione elettorale che poneva stabilmente i partiti di sinistra, a cominciare da quello comunista, al centro di un vasto tessuto di relazioni politiche, sociali e professionali (Galli 1968; Ramella 2005). Il ruolo predominante del PCI in città ha condizionato, per almeno un cinquantennio, l'intera politica bolognese, garantendo stabilità governativa, continuità nelle politiche pubbliche e un livello decisamente elevato di prevedibilità elettorale, sia dal lato dei cittadini che da quello dei partiti. Ovviamente, questo scenario politico ha per molto tempo impedito l'avverarsi dell'alternanza al potere, vale a dire la possibilità di sostituire un'intera classe dirigente cittadina con l'ingresso di rappresentanti di altri partiti di centro o di destra.

Questo scenario di immobilità politica, se non di immobilismo, incomincia a cambiare abbastanza radicalmente a partire dai primi anni Novanta, sia per ragioni di tipo storico e geopolitico sia, e forse ancora di più, per motivi interni o endogeni. Tra questi ultimi, assunsero un carattere cruciale i cambiamenti intervenuti nella forma di governo municipale (di impianto neo-parlamentare) e nel sistema elettorale che prevedeva l'elezione diretta del sindaco (Baldini e Legnante 2000; Caciagli e Di Virgilio 2004). Come vedremo, questi nuovi meccanismi istituzionali ed elettorali hanno profondamente cambiato il formato e la meccanica del sistema partitico bolognese, rendendo un po' meno prevedibile e decisamente più stabile la politica all'interno dell'arena esecutiva.

In concomitanza con le - o forse anche come conseguenza delle - trasformazioni introdotte nell'architettura istituzionale, abbiamo assistito altresì a profondi mutamenti intervenuti negli orientamenti di voto dei cittadini bolognesi e nelle strutture organizzative dei partiti. Le campagne elettorali si sono fatte più personalizzate, la competizione politica si è progressivamente bipolarizzata, la partecipazione al voto è andata sensibilmente riducendosi, gli esiti del voto sono diventati sempre meno prevedibili e, alla fine, il modello politico bolognese ha perso quei tratti tipici che lo rendevano eccezionale rispetto a altre zone d'Italia. Nel corso dell'ultimo ventennio di elezioni municipali, la città di Bologna si è progressivamente "normalizzata", allineandosi alle trasformazioni intervenute nel frattempo nelle altre grandi città e anche nel sistema politico italiano nel suo complesso. 
Non ci sono, però, ancora analisi o studi che cerchino di tracciare un bilancio dei mutamenti avvenuti a Bologna dagli anni Novanta fino ad oggi. La maggior parte delle ricerche si concentra su un'unica tornata elettorale, magari altamente significativa (come quella "storica" del 1999), o su determinati episodi o attori politici di rilievo. Manca uno studio longitudinale che riesca a tenere assieme l'intero ciclo elettorale che si è aperto nel 1995, con il primo sindaco eletto direttamente dai cittadini, e si è concluso nella primavera nel 2016 (vedi tab. 1). Questo articolo ha il preciso scopo di colmare questa lacuna e lo fa analizzando le trasformazioni della politica bolognese sia dal lato del comportamento degli elettori sia da quello dell'offerta partitica. Più precisamente, dopo una breve cronistoria delle sei elezioni che si sono tenute a Bologna dalla metà degli anni Novanta fino ad oggi, il terzo paragrafo si concentrerà sull'analisi dei flussi elettorali per capire se e in che misura l'elettorato bolognese sia diventato nel corso del tempo più mobile ovvero disposto a cambiare le proprie preferenze in base alle proposte politiche in campo. Diversamente, nel paragrafo quarto l'attenzione verrà rivolta sulla struttura dell'offerta partitica, per comprendere se ci siano stati cambiamenti di rilievo e, soprattutto, se siano stati in grado di modificare i rapporti di competizione e collaborazione tra i partiti politici. Infine, l'ultimo paragrafo sarà dedicato ad un'analisi ad ampio raggio dello scenario politico-elettorale, cercando di mettere il caso di Bologna in una prospettiva storica di lungo periodo. È probabile, infatti, che con le elezioni amministrative del 2016 si sia chiuso un ciclo politico e, al contempo, stia prendendo forma, in modo lento e disordinato, una nuova fase caratterizzata da fluidità elettorale e multipolarismo partitico. Per provare a immaginare quello che succederà in futuro, bisogna avere un quadro molto chiaro di quello che è successo fino ad oggi. Questo è l'obiettivo che ci siamo prefissati di raggiungere.

\section{TAB. 1 - Risultati delle elezioni comunali a Bologna dal 1995 al 2016.}

\begin{tabular}{|c|c|c|c|c|c|c|}
\hline \multirow[b]{2}{*}{ Elezione } & \multicolumn{3}{|c|}{ Candidato vincitore } & \multicolumn{3}{|c|}{ Principale sfidante ( $2^{\circ}$ classificato $)$} \\
\hline & Nome & N. voti assoluti & $\%$ voti & Nome & N. voti assoluti & $\%$ voti \\
\hline $\begin{array}{l}1995 \\
1^{\circ} \text { turno }\end{array}$ & W. Vitali & 145.664 & 50,4 & $\begin{array}{l}\text { G. Gazzoni } \\
\text { Frascara }\end{array}$ & 48.615 & 16,8 \\
\hline $\begin{array}{l}1999 \\
1^{\circ} \text { turno }\end{array}$ & G. Guazzaloca & 104.565 & 41,5 & S. Bartolini & 117.367 & 46,6 \\
\hline 1999 Ballottaggio & G. Guazzaloca & 113.462 & 50,7 & S. Bartolini & 110.390 & 49,3 \\
\hline $\begin{array}{l}2004 \\
1^{\circ} \text { turno }\end{array}$ & S. Cofferati & 140.795 & 55,9 & G.Guazzaloca & 102.221 & 40,6 \\
\hline $\begin{array}{l}2009 \\
1^{\circ} \text { turno }\end{array}$ & F. Delbono & 112.127 & 49,4 & A. Cazzola & 66.056 & 29,1 \\
\hline 2009 Ballottaggio & F. Delbono & 112.789 & 60,8 & A. Cazzola & 72.798 & 39,2 \\
\hline $\begin{array}{l}2011 \\
1^{\circ} \text { turno }\end{array}$ & V. Merola & 106.070 & 50,5 & M. Bernardini & 63.799 & 30.4 \\
\hline $\begin{array}{l}2016 \\
1^{\circ} \text { turno }\end{array}$ & V. Merola & 68.772 & 39,5 & M. Bernardini & 38.807 & 22,3 \\
\hline 2016 Ballottaggio & V. Merola & 83.907 & 54,6 & L. Borgonzoni & 69.660 & 45,4 \\
\hline
\end{tabular}

Fonte: Ministero dell'Interno. 


\section{L'offerta partitica nelle elezioni comunali bolognesi}

Le elezioni del 1995. - Il debutto dell'elezione diretta del sindaco nella città di Bologna, cuore e simbolo della cosiddetta subcultura rossa, avviene nel 1995. Come da tradizione, nonostante le innovazioni dal lato istituzionale, il risultato sul piano politico non riservò particolari sorprese. Il sindaco uscente (Walter Vitali), proveniente dal Partito Comunista Italiano (PCI), aveva deciso di presentarsi alle elezioni come candidato unitario dell'intero schieramento di centrosinistra, comprendente una parte di ex-democristiani (Democratici per Bologna) e i Verdi. Il principale sfidante di Vitali fu Filippo Berselli, appena eletto senatore (nel 1994) nelle fila di Alleanza Nazionale. A suo sostegno, oltre al partito erede del Movimento sociale italiano, si era schierata anche la lista Unione federalista, un piccolo ed effimero partito fondato dal teorico della politica Gianfranco Miglio. Per Forza Italia, decise di scendere in campo l'imprenditore Giuseppe Gazzoni Frascara, da poco divenuto anche presidente della squadra di calcio della città bolognese.

Gli altri candidati erano, in parte, espressione delle forze più estreme dello spazio politico oppure esponenti di partiti minori o di movimenti cittadini, di natura prevalentemente civica. Il giovane Partito della Rifondazione Comunista si presentava alle elezioni con Ugo Boghetta, parlamentare dal 1992 al 2001, mentre la Lega Nord, dopo la prima esperienza a Bologna nel 1990, decise di presentarsi nuovamente alle elezioni municipali guidata da Luigi Pasquini. A completare il quadro si aggiunsero Carlo Monaco, docente di Filosofia e candidato per la Lista Pannella-Riformatori, l'ingegnere Giovanni Salizzoni per la lista civica "Governare Bologna", Marcantonio Bezicheri come esponente del Movimento Sociale-Fiamma Tricolore e, per finire, due candidati civici o non-partitici: Ivanoe Stefanelli ("È tempo di cambiare") e Aldo Dinacci ("Movimento Padri Separati").

Nonostante l'estrema frammentazione partitica, frutto di un sistema dei partiti già in fase di destrutturazione, la coalizione di centrosinistra riuscì a confermare la propria vittoria e, soprattutto, ad evitare per un soffio il ballottaggio. Vitali raccolse, infatti, il $50,4 \%$ dei consensi e, grazie al premio di maggioranza previsto dalla nuova normativa elettorale, ottenne i due terzi dei seggi nel consiglio comunale (28 su 42). Un'esigua maggioranza di voti aveva consentito ai partiti del centrosinistra di confermare e perpetuare il proprio predominio elettorale in ambito cittadino, anche perché lo schieramento opposto era diviso e frazionato al suo interno. Detto altrimenti, la vittoria del centrosinistra a Bologna nel 1995 fu soprattutto una "vittoria per mobilitazione", derivante dalla capacità dei partiti eredi del PCI di riportare alle urne il proprio elettorato ancora radicato e di appartenenza. Non fu, certo, una vittoria di competizione, combattuta sul filo dei voti nei confronti di un'opposizione sfilacciata e in buona misura disorganizzata. Per la precisione, nel campo del centrodestra, Berselli ottenne il 18\% dei voti e Gazzoni il 16,8\%, dividendosi un bottino elettorale che poteva essere capitalizzato e reso più competitivo sotto la guida di un unico leader.

Con le elezioni del 1995, secondo molti studiosi e commentatori, si chiudeva un intero ciclo politico che si era aperto con la sindacatura di Vitali nel 1990 e che, sotto diversi punti di vista, poteva essere interpretato come la parabola conclusiva di una classe dirigente locale che, con il crollo repentino del Muro di Berlino, non aveva saputo ade- 
guarsi a nuovi tempi della politica post-ideologica. Si aprì così una fase del tutto nuova nella storia elettorale bolognese, dove tutto (o quasi) poteva succedere.

Le elezioni del 1999. - Con le elezioni del 1999 avvenne nella città di Bologna, e cioè nella principale vetrina del buongoverno (post)comunista a livello comunale, la prima, storica, quasi del tutto inattesa alternanza. Come abbiamo già indicato in precedenza, quella tornata elettorale si inseriva nella fase discendente di un'intera classe dirigente locale, che aveva perso la capacità di elaborazione progettuale per il futuro della città e si accontentava della rendita di governo prodotta dalle passate amministrazioni. In una prospettiva analitica, è possibile osservare che le elezioni del 1999 rappresentarono, per la sinistra bolognese, un momento di passaggio - sicuramente traumatico - da un sistema di governo dominato dal partito ad un sistema in cui cominciava a farsi strada la figura di un leader al di sopra del proprio schieramento. Questa trasformazione, che era il frutto sia dei mutamenti intervenuti all'interno del principale partito della sinistra in Italia sia del nuovo sistema di elezione per il sindaco introdotto in Italia a partire dal 1993, aveva avuto evidenti ripercussioni anche nel contesto bolognese.

Come da tipica tradizione gramsciana, nel 1999 arrivò a Bologna una situazione di crisi dove «il vecchio muore e il nuovo non può nascere» (Gramsci 1975). Nel caso specifico, il "vecchio" che stava scomparendo era il classico party government in salsa emiliana, il cui perno era rappresentato da un partito di massa, radicato sul territorio e ramificato all'interno delle principali strutture associative (dai sindacati alle cooperative, passando per le strutture ricreative fino a raggiungere pezzi del mondo imprenditoriale). Quel mondo e, soprattutto, quel partito non c'erano più: erano stati travolti dal crollo del Muro di Berlino e, più in generale, da un progressivo processo di secolarizzazione e modernizzazione della società italiana. Insomma, il "vecchio" era scomparso. Tuttavia, il nuovo faticava a farsi strada, nonostante fosse incentivato dalle nuove regole elettorali che cercavano di mettere al centro dell'azione politica le istituzioni di governo e, in particolare, il vertice dell'esecutivo (Baldini, Corbetta e Vassallo 2000; Pasquino 2011).

Nel caso di Bologna, il PDS/DS rimase intrappolato in questa fase di transizione da un vecchio a un nuovo modello di governo. A farne le conseguenze fu, in prima battuta, il sindaco uscente Vitali e, successivamente, l'intero schieramento di centrosinistra. Nell'individuazione della candidatura all'interno dei DS si aprì quella che correttamente alcuni studiosi definirono una "guerra di logoramento» (Baldini, Corbetta e Vassallo 2000), e cioè un tentativo (poi riuscito) di indebolimento della leadership di Vitali allo scopo di riacquisire posizioni di vantaggio da parte della dirigenza post-comunista. $\mathrm{Fu}$ da questa situazione di incertezza complessiva che dal centrosinistra emerse la proposta di utilizzare - per la prima volta nel contesto cittadino - le elezioni primarie per l'individuazione del candidato sindaco (Sandri e Valbruzzi 2012). Dopo vari tentennamenti e, soprattutto, dopo il gran rifiuto a prendere parte alla competizione da parte di Mauro Zani (esponente di spicco della storica dirigenza del PCI), la coalizione guidata dai DS decise di organizzare elezioni primarie per stabilire il futuro candidato sindaco.

Gli eventi che condussero alle prime primarie bolognesi cominciarono tra la fine del 1998 e i primi mesi del 1999, ma i prodromi potevano già essere individuati «nella 
lacerante contesa per la segreteria del '96» (Anderlini 2006, p. 136) all'interno del partito erede del PCI, ininterrottamente al governo della città per oltre cinquanta anni, da Giuseppe Dozza a Walter Vitali. È in quel biennio, in particolare tra l'autunno del 1998 e l'inverno del 1999 che si consumò una crisi acuta, in parte, ma non soltanto, di successione, all'interno dei DS, innescata soprattutto dall'insoddisfacente operato dell'allora sindaco in carica, Vitali, e dalle ambizioni del segretario cittadino del partito, Alessandro Ramazza. Un «guerra civile», per usare ancora le parole del sociologo Anderlini (2006, p. 136), conclusa soltanto qualche mese più tardi mediante il "commissariamento" affidato a dirigenti inviati direttamente da Roma, e che, però, nascondeva molto di più di una semplice competizione interna per la carica di sindaco. Come anticipato, dietro quella contesa si intravedeva già allora la crisi di un partito che aveva perso, dapprima, la sua chiara identità e, di seguito, la sua centralità all'interno di una rete di relazioni - politiche, sociali, professionali - sulla quale il modello bolognese (ed emiliano più in generale) si era sviluppato e rafforzato. Il lato più importante di quel «triangolo virtuoso» (Pasquino 2011, p. 65) composto da un grande partito perennemente al governo, un grande e robusto sindacato, e un ampio conglomerato cooperativo, si stava spezzando, trascinando dietro di sé l'intera, preziosa costruzione. Un primo modo, alquanto improvvisato, per cercare di rimettere assieme $\mathrm{i}$ cocci di un partito dilaniato dai dissidi, fu di organizzare in fretta delle primarie "sotto tutela". Nel marzo del 1999, dunque, la Quercia - più qualche cespuglio di contorno - sperimentò primarie di coalizione che ebbero uno scarso successo in termini di mobilitazione e una vincitrice predestinata, Silvia Bartolini.

Il finale della storia è ormai più che noto: Bologna sperimentò nel 1999 la sua prima, inaspettata, per alcuni anche traumatica, alternanza di governo (Campus e Pasquino 2000; Baldini, Corbetta e Vassallo 2000). Il modello neo-corporativo in scala locale, "partito-sindacato-industriali", si era definitivamente incrinato e l'artefice di quella sconfitta fu un candidato civico, che proveniva dal mondo associativo locale (come presidente della Confcommercio bolognese) e che riuscì a compattare attorno alla sua figura le principali forze del centrodestra (ad eccezione della Lega Nord, ancora in versione "autonomista"). La vittoria per Guazzaloca arrivò al secondo turno, mentre nel primo round della competizione risultò in testa la candidata del centrosinistra che raccolse il 46,6\% dei consensi (rispetto al $41,5 \%$ in favore di Guazzaloca). Nel ballottaggio si capovolsero totalmente i ruoli e la candidatura civica del centrodestra prevalse di misura $(50,7 \%) \mathrm{su}$ quella del centrosinistra (49,3\%).

Come è stato notato, l'alternanza del 1999 dipese «in gran parte da un giudizio negativo degli elettori su quanto i politici locali avevano fatto (e non fatto) nel quinquennio precedente - piuttosto che per la lunga permanenza al potere» (Baldini, Corbetta e Vassallo 2000, p. 242). Tuttavia, l'esito di quella elezione segnalava la conclusione definitiva di un certo modo di intendere il governo cittadino e anche di gestire un partito che non era più quello idealtipico, e un po' idealizzato, degli anni Sessanta e Settanta. Quella pagina di storia era stata definitivamente chiusa.

Le elezioni del 2004. - L'alternanza sperimentata nel 1999 si rivelò un evento isolato, non in grado di aprire un nuovo ciclo elettorale basato sull'equilibrio nella competizione tra 
i due maggiori poli. Per l'intero quinquennio dell'amministrazione Guazzaloca, l'atmosfera politica a Bologna era quella di una coabitazione informale forzata e maldigerita, con il centrosinistra pronto a tutto pur di riconquistare lo scettro del potere cittadino. Alla prima occasione utile, lo schieramento all'opposizione riconquistò il governo della città grazie alla candidatura di quello che allora venne definito un "briscolone" nazionale, ossia Sergio Cofferati, segretario generale della CGIL fino al 2002 e alla ricerca di un'arena politica dove mettere alla prova le sue doti di leadership. Se nel 1999 i DS erano ancora intrappolati nella fase transitoria da un modello di governo partito-centrico ad uno maggiormente incentrato sulle istituzioni governative e sul leader, con l'arrivo di Cofferati quella transizione poteva considerarsi conclusa. Per di più, questa soluzione, calata dall'esterno, risolveva molti dei problemi dei partiti di centrosinistra sotto le Due Torri, e non solo. A livello nazionale veniva "sistemato" un concorrente potenzialmente scomodo per la corsa alla leadership nazionale contro Berlusconi, mentre a livello locale la figura di Cofferati rappresentava non solo una garanzia di vittoria, ma anche la possibilità di nascondere sotto il tappeto tutti i ritardi, i problemi e i difetti di un apparato partitico che, neppure dall'opposizione, era riuscito a riorganizzarsi e rimodellarsi.

Naturalmente, almeno per i dirigenti dei DS, le leadership di Cofferati non aveva alcun bisogno di essere rodata nel fuoco delle primarie, e infatti venne recepita, sostanzialmente, a scatola chiusa. Forse per la prima volta nel contesto bolognese, dove da sempre l'apparato del partito aveva dominato e controllato la nascita di leadership locali, si assistette, nell'ambito del centrosinistra, ad una campagna elettorale fortemente personalizzata sulla figura di Cofferati (Grandi e Vaccari 2004), il candidato che avrebbe permesso agli eredi del PCI di tornare ad amministrare la città.

Dall'altra parte, veniva confermata la candidatura civica di Giorgio Guazzaloca che, oltre che da una sua lista personale (la più votata all'interno dello schieramento: $18,4 \%$ dei voti), era appoggiato da Forza Italia, Alleanza Nazionale e da una lista minore di centrodestra. Nonostante ci fossero altre sette candidature minori, tra cui quelle della Lega Nord (con Simone Albertini) e del Nuovi PSI (a sostegno del professor Franco Piro), la competizione si concentrò in larga misura sulle due coalizioni principali. Infatti, le due candidature raccolsero congiuntamente oltre il $96 \%$ dei voti: un indicatore della spiccata bipolarizzazione della contesa politica bolognese. A prevalere, senza necessita di ballottaggio, fu Sergio Cofferati con il 55,9\% dei consensi, mentre il sindaco uscente si fermò al 40,6\%. Dopo la sconfitta inattesa del 1999, Bologna "la rossa" tornò quindi ad essere amministrata dal centrosinistra, grazie ad una candidatura esterna, proveniente cioè dal di fuori dell'apparato partitico cittadino: una eventualità de tutto impensabile fino a qualche anno prima.

Le elezioni del 2009. - Convinto che non si potesse essere «padre a Genova e sindaco a Bologna», Cofferati decise di non ricandidarsi alle successive elezioni del 2009, lasciando il centrosinistra locale senza un candidato, ma con molte polemiche al suo interno. In realtà, l'amministrazione di Cofferati, che aveva suscitato numerose aspettative fin dal suo inizio, non si rivelò né particolarmente brillante, né particolarmente innovativa. $\mathrm{Su}$ molti aspetti, soprattutto sulle grandi opere infrastrutturali e sulle questioni sociali, erano 
emerse opinioni diverse e distanti tra il sindaco e gli organi dirigenti del partito bolognese (ormai trasformatosi in Partito Democratico). Sia per i cittadini che per una parte del PD, l'esperienza di Cofferati era stata vissuta come una sorta di "ingerenza esterna" e lo stesso sindaco non era mai riuscito a entrare pienamente in sintonia con la città. Alla fine, il divorzio fu consensuale: Cofferati avrebbe proseguito la sua vita privata (e politica) a Genova e i dirigenti locali del PD sarebbero tornati in controllo dell'apparato amministrativo. Tuttavia, in assenza di un leader "naturale", la coalizione di centrosinistra decise di scegliere il proprio candidato ricorrendo alle elezioni primarie, che furono vinte da Flavio Delbono, il candidato sostenuto e ampiamente sponsorizzato dai leader del PD, locali e nazionali (Seddone e Valbruzzi 2010).

Nel centrodestra, invece, prese forma la candidatura di Alfredo Cazzola, un imprenditore molto noto in città (anche per essere stato presidente del Motor Show, della Virtus Pallacanestro e del Bologna F.C.) che riuscì, per la prima volta nella storia del capoluogo emiliano-romagnolo, a costruire un'alleanza di centrodestra ampia, includente anche la Lega Nord. A "rovinare" quest'immagine di unità, contribuì però la candidatura civica portata avanti da Giorgio Guazzaloca, il quale decise di correre come indipendente staccato da ogni etichetta di partito. A completare il quadro, oltre alla presenza di due candidati sindaci che si muovevano nell'ambito della sinistra più (Valerio Monteventi) o meno (Gianfranco Pasquino) radicale, si inserì per la prima volta la candidatura di un esponente del Movimento 5 Stelle: il ventottenne Giovanni Favia.

Nel 2009 l'offerta partitica si presentava, dunque, particolarmente frammentata e già si intravedevano i germi di un multipolarismo che si sarebbe poi progressivamente affermato anche in chiave nazionale. Rispetto a quanto accaduto nella tornata precedente, la coalizione di centrosinistra guidata da Delbono, fermandosi al 49,4\% dei voti, non riuscì a conquistare la vittoria al primo turno. Servì il ballottaggio a decretare la sconfitta dello schieramento guidato da Cazzola $(39,2 \%)$ e la definitiva (ma temporanea) affermazione di Delbono $(60,8 \%)$.

Come anticipato, le elezioni bolognesi del 2009 videro il debutto del Movimento 5 Stelle a livello municipale. Con la candidatura di Giovanni Favia, il M5S si rivelò fin da subito capace di attrarre consensi in modo trasversale, anche se la candidatura pentastellata si sarebbe fermata al 3,3\% dei voti.

In generale, le elezioni del 2009 rappresentarono una temporanea conferma per la coalizione di centrosinistra, la quale riuscì a vincere (ma soltanto al secondo turno) soprattutto grazie alle divisioni nello schieramento di centrodestra, mostrando comunque alcuni segni di cedimento nel proprio classico elettorato di riferimento. Tuttavia, $i$ problemi maggiori per la nuova amministrazione di Delbono cominciarono all'indomani delle elezioni, quando il sindaco stesso venne prima indagato e poi condannato per abuso d'ufficio e peculato. A quel punto si aprì una delle stagioni più tristi nella storia di Bologna: da sempre considerata un modello di civismo e buona politica, si trovò in brevissimo tempo commissariata dal Ministero dell'Interno e affidata, per l'ordinaria amministrazione, al prefetto Annamaria Cancellieri (fino al maggio del 2011). Il mito del buongoverno bolognese si era ormai gravemente incrinato, così come la fiducia dei cittadini nei confronti della politica e dei partiti. 
Le elezioni del 2011. - Dopo il breve, ma significativo, interludio del commissariamento cittadino, le elezioni anticipate erano state fissate per il maggio 2011. Di fronte al clamoroso fallimento del centrosinistra con Delbono, molti gruppi politici alternativi videro aprirsi grandi finestre di opportunità. In molti, cioè, pensarono si potessero ricreare le condizioni che avevano portato alla storica alternanza del 1999. Questa sensazione di precarietà e incertezza incentivò una ulteriore frammentazione dell'offerta partitica, soprattutto nell'ambito del cosiddetto civismo bolognese. Ovviamente, l'operazione più difficile spettava allo schieramento di centrosinistra, il quale aveva il compito di far dimenticare al proprio elettorato l'incidente del 2009. Anche in questo caso, per aprire ulteriori spazi di partecipazione e provare a mobilitare gli elettori in vista del voto, la coalizione guidata dal PD si affidò allo strumento delle primarie. A differenza del passato, le elezioni primarie del 2011 non avevano un vincitore preannunciato, definito e protetto ex ante grazie agli accordi stretti tra i principali dirigenti di partito. Nella competizione interna al centrosinistra, aperta a tutti gli iscritti e simpatizzanti dei partiti che componevano la coalizione (da SEL all'Italia dei Valori), risultò vincitore Virginio Merola, all'epoca presidente del consiglio provinciale di Bologna ed ex assessore all'Urbanistica nella giunta di Cofferati. Senza troppi traumi e con un buon livello di partecipazione, il centrosinistra aveva, dunque, scelto il suo candidato: un funzionario di partito senza grandi doti comunicative, ma in grado di mantenere stabili gli equilibri interni al PD e alla sua coalizione più in generale.

Dall'altra parte, facendo tesoro dell'esperienza unificante di Cazzola nel 2009, il centrodestra si presentò coeso, con il Popolo della Libertà e la Lega Nord a sostegno di Manes Bernardini, esponente leghista in ambito cittadino. L'unica componente assente all'interno di questo quadro unitario fu quella centrista dell'UDC, che preferì appoggiare la candidatura civica di Stefano Aldrovandi, noto amministratore e imprenditore bolognese.

Dopo il positivo debutto del 2009, anche il M5S decise di ripresentarsi alle elezioni: questa volta candidando un rappresentante molto vicino all'ala più "pura" del Movimento e componente del Direttorio pentastellato. La scelta di Massimo Bugani si rivelò corretta e, infatti, i voti per il M5S triplicarono nel giro di un biennio, passando dal 3,3\% al 9,5\%. Oltre alla lista grillina, si aggiunsero anche altre liste minori, tra le quali quella indipendente di Daniele Corticelli, quella del Partito comunista dei lavoratori (con Michele Terra) e due di estrema destra (Elisabetta Avanzi per Forza Nuova e Anna Montella per La Destra).

Le elezioni anticipate si tennero il 15 maggio 2011 e furono segnate da un'offerta partitica alquanto frammentata e da un'incertezza di fondo sull'esito elettorale. In particolare, l'incertezza riguardava l'eventualità di un secondo turno che avrebbe potuto favorire il ricompattamento dell'intero elettorato di centrodestra e, quindi, la possibilità di una nuova alternanza di governo. Gli elettori bolognesi decisero però di chiudere la partita direttamente al primo turno, consegnando a Merola il 50,5\% dei voti, mentre il centrodestra si fermò al 30,4\%: una vittoria che consentì al centrosinistra di evitare per un pugno di voti il ballottaggio e, soprattutto, di riconquistare il governo della città dopo il trauma del commissariamento sperimentato nel 2011. Un risultato tutt'altro che scontato. 
Le elezioni del 2016. - Alla vigilia della tornata elettorale del 2016 erano in pochi, e forse nessuno, a prevedere uno scenario politico "aperto", cioè elettoralmente competitivo e incerto. La maggior parte degli opinionisti e dei sondaggisti dava per acquisita la vittoria di Virginio Merola, sindaco uscente espressione del PD, già a partire dal primo turno. Insomma, anche questa volta non ci sarebbero state sorprese in quella che, per molto tempo, è stata considerata la città-simbolo, il laboratorio o, addirittura, il modello del "socialismo municipale" che doveva servire a legittimare, se non a rafforzare, la prospettiva di una scalata al governo nazionale per gli esponenti di quello che era il PCI (Anderlini 2006).

Invece, le elezioni del giugno 2016 hanno riservato diverse sorprese. Innanzitutto, chi pensava che Merola avesse di fronte a sé una strada in discesa verso il secondo mandato si è dovuto prontamente ricredere dopo il risultato per nulla decisivo del primo turno. La strada in discesa si è rivelata più in salita del previsto, anche perché la coalizione di centrosinistra si è fermata al di sotto del $40 \%$ dei consensi nel primo turno di votazione. Un risultato non solo inatteso ma deludente per il sindaco uscente e per il PD, la cui ricandidatura era stata variamente messa in discussione nel corso dell'intera campagna elettorale.

Un'altra sorpresa è arrivata dalla candidata del centrodestra che a Bologna si presentava, senza troppa enfasi, compattamente a sostegno della candidata leghista Lucia Borgonzoni, promossa leader cittadina della Lega Nord direttamente da Matteo Salvinie capace, dopo molti tentennamenti, di aggregare il fronte ampio dell'intero centrodestra, dalla Lega fino ai Fratelli d'Italia di Giorgia Meloni, passando ovviamente per Forza Italia.

A suo modo sorprendente è stato anche il risultato ottenuto dal candidato del Movimento 5 Stelle, Massimo Bugani, una personalità "discussa" fuori e dentro il circuito pentastellato, ma in strettissimi rapporti sia con il Direttorio del non-partito sia con il centro webcratico del Movimento (Beppe Grillo e gli esponenti dalla Casaleggio e associati). Di fronte a vere e proprie sorprese elettorali in altre realtà cittadine, come quelle di Chiara Appendino a Torino (Cepernich e Vignati 2016) e Virginia Raggi a Roma (Natalizia e Vignati 2016), il risultato di Bologna per il M5S ha avuto un sapore agrodolce: positivo se osservato in modo diacronico nella storia della città felsinea (dal 9,5\% del $2011 \mathrm{al}$ $16,6 \%$ del 2016), negativo se paragonato sincronicamente ad altri exploit dei pentastellati in chiave nazionale.

Per ciò che riguarda l'esito generale del voto è importante osservare innanzitutto la partecipazione elettorale che è il dato più importante per capire e provare a stimare in che misura la disaffezione politica abbia colpito i cittadini, in particolare nel rapporto che li lega ai loro rappresentanti nelle istituzioni. In questa prospettiva, il caso di Bologna è particolarmente rilevante per due ragioni. La prima è legata al ruolo della città, capace di riassumere in sé tutte le principali caratteristiche della subcultura politica territoriale tipica delle regioni saldamente controllate dai partiti di sinistra, e cioè una diffusa, frequente, stabile partecipazione alle attività politico-associative. Il voto è sempre stato l'espressione suprema di questo robusto sentimento civico, la punta di un iceberg che aveva solide radici nel ricco capitale sociale che caratterizza(va) le regioni dell'Italia centrale (Putnam et al. 1993; Cartocci 2007; Vassallo 2013). La seconda ragione rimanda, invece, ai risultati delle elezioni regionali del 2014, quando in tutta l'Emilia-Romagna si recarono ai seggi meno di quattro elettori su dieci e a Bologna l'affluenza si fermò al 39,7\%. Un dato 
preoccupante in una ragione tradizionalmente molto partecipativa e che indicava, forse per la prima volta, il venir meno di uno degli elementi portanti della subcultura politica: la disponibilità al voto, la volontà di prendere parte al gioco democratico per eccellenza.

Come mostra la figura 1, il dato delle ultime regionali a Bologna era stato il più basso mai registrato in città dal 1994, con un calo di quasi 30 punti percentuali rispetto alle votazioni precedenti. All'interno di questo scenario e di fronte a un crollo così netto della partecipazione elettorale, le elezioni comunali del 2016 lasciavano poco spazio alle speranze del centrosinistra. Il massimo che ci si poteva aspettare era un crollo meno marcato in confronto a quello osservato nel 2014 per l'elezione del presidente della regione. E così effettivamente è stato. Rispetto alle comunali del 2011, quando si erano recate ai seggi oltre 21 mila persone (pari al $71,4 \%$ del corpo elettorale), la partecipazione nel 2016 è calata di quasi 12 punti percentuali, attestandosi al 59,7\%. Un calo superiore sia a quello medio osservato in tutti gli altri comuni andati al voto in Italia in questa tornata elettorale (-5,5 punti percentuali) sia a quello dei soli comuni emiliano-romagnoli $(-8,4$ punti percentuali). Peraltro, il dato di Bologna del 2016 è il più basso nell'intera storia delle comunali della città e segnala - ma in realtà accelera - un trend discendente che era già cominciato a partire dalla metà degli anni 2000, quando la partecipazione elettorale superava ancora 1 ' $80 \%$.

FIG. 1 - Partecipazione elettorale nel comune di Bologna dal 1994 al 2016 (\% di votanti su elettori).

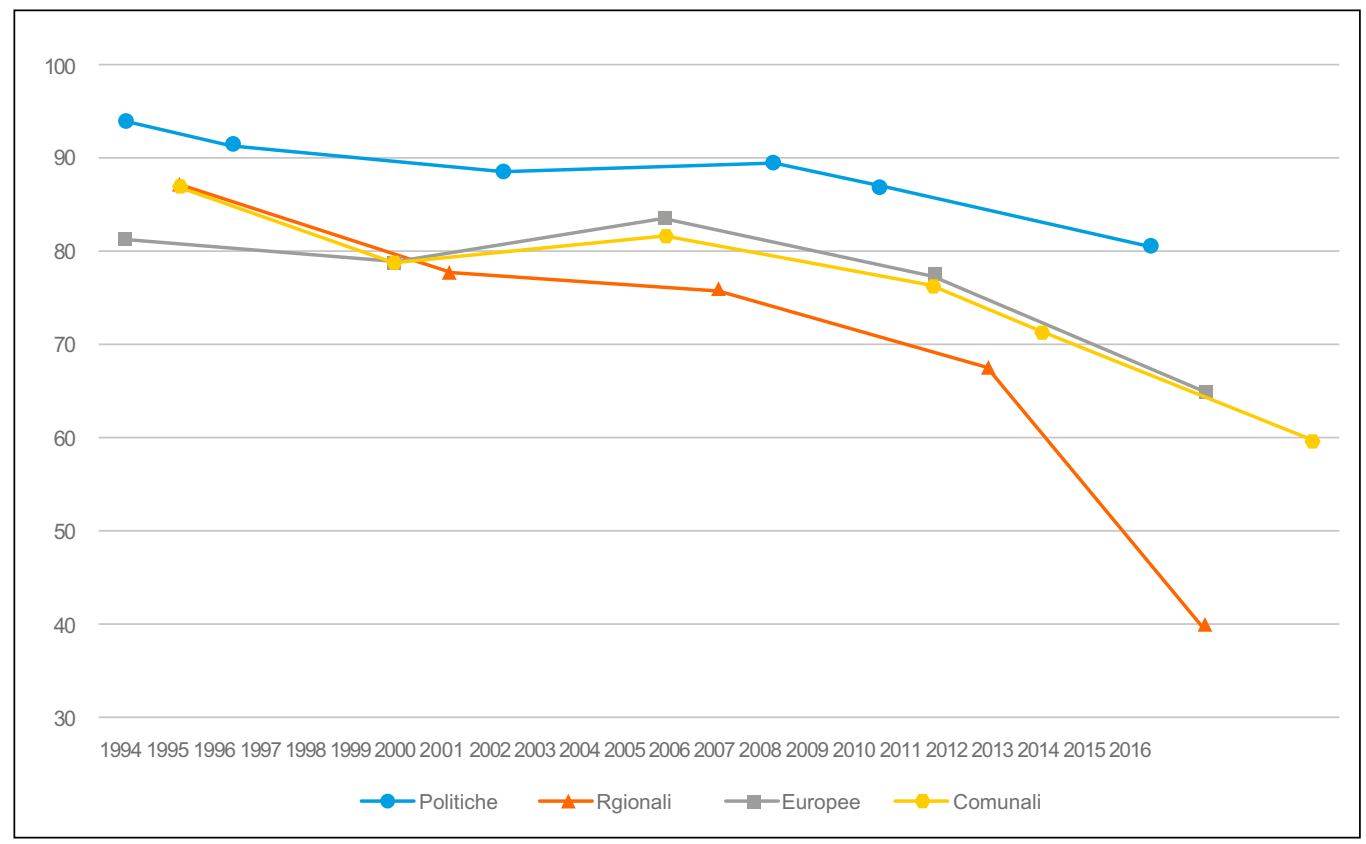

Fonte: Nostra elaborazione su dati del Ministero dell'Interno. 
Ciò detto, possiamo passare ad analizzare gli esiti del voto per capire quali partiti hanno perso o preso voti rispetto alle votazioni precedenti.

Il primo dato da evidenziare, probabilmente il più importante, è, si è detto, che la coalizione di centrosinistra guidata dal sindaco uscente Merola non è riuscita a vincere già al primo turno. Nel 2011 Merola aveva ottenuto il 50,5\% dei voti, diventando così sindaco senza dover ricorrere al ballottaggio. Nel 2016, invece, il centrosinistra non ha superato al primo turno il $40 \%$ dei voti, perdendo esattamente 11 punti percentuali di consenso. Il secondo dato rilevante è la distribuzione del voto tra i partiti che si sono sottoposti al giudizio degli elettori. Se nel 2011 (così come nelle elezioni precedenti) la struttura della competizione inter-partitica, per quanto in modo «imperfetto», era bipolare, tra un polo di centrosinistra e uno di centrodestra, le elezioni del 2016 certificano anche a livello comunale quello che si era già palesato a livello nazionale nel 2013, ossia l'avvento del tripolarismo (Chiaramonte e Emanuele 2013; Bolgherini e Grimaldi 2015; Tronconi 2015).

Cinque anni prima, i due principali contendenti raccoglievano assieme quasi 1' $81 \%$ dei voti, mentre oggi i tre candidati più votati (Merola, Borgonzoni e Bugani) controllano complessivamente soltanto il 78,4\% dei consensi. Questo significa, da un lato, che lo "schema di gioco" è ormai diventato tripolare anche a livello municipale e che, dall'altro lato, è aumentata la frammentazione politica e la forza dei candidati "altri" o relativamente minori.

Il terzo ed ultimo dato rilevante riguarda il ballottaggio e, soprattutto, la capacità del centrodestra di allargarsi nel corso dei due turni di votazione. Lucia Borgonzoni passa, infatti, dal $22,3 \%$ del primo turno al $45,4 \%$, raddoppiando i propri consensi $(+30.853$ voti) e dimostrando una notevole capacità di espansione elettorale. Tuttavia, questa significativa "elasticità" del centrodestra nel 2016 non è riuscita ad ostacolare la rielezione del sindaco uscente Merola, il quale, con il 54,6\% dei voti (+15.135 in termini assoluti) ha vinto il ballottaggio e superato di 10 punti percentuali la sua avversaria. Cruciale è stata, dunque, l'abilità della coalizione di centrosinistra nel mobilitare il proprio elettorato, anche in vista del secondo turno, evitando così una sconfitta per smobilitazione come era avvenuto, invece, nel 1999.

In sintesi, il risultato bolognese delle comunali del giugno 2016 ha offerto molte più sorprese del previsto $\mathrm{e}$, soprattutto, ha definitivamente rotto qualche logoro tabù. Chi pensava a Bologna come ad una città ancora elettoralmente immobile e non contendibile, e cioè impermeabile rispetto alle proposte politiche di altri partiti che non fossero quelli del centrosinistra, si è dovuto ricredere. Le elezioni del 2016 sanciscono ormai anche per Bologna l'ingresso nell'era della contendibilità politica (Bartolini 1996): nulla, neppure la rielezione di un sindaco targato $\mathrm{PD}$, può essere dato per scontato e la stabilità - per non dire la fissità - del comportamento elettorale dei cittadini bolognesi, come vedremo nel prossimo paragrafo, è soltanto un lontano ricordo. L'intero ciclo elettorale che si è aperto nel 1995 con la prima elezione diretta del sindaco può essere interpretato, infatti, come un progressivo processo di "scongelamento" delle fratture sociali e, soprattutto, delle abitudini elettorali che avevano caratterizzato il voto dei cittadini bolognesi nell'intero cinquantennio del secondo dopoguerra. Il predominio politico del centrosinistra a Bologna si è fatto sempre più debole e ogni tornata elettorale ha introdotto nuovi elementi di incertezza. 
In questa prospettiva di lungo periodo, le ultime elezioni amministrative segnano sicuramente l'apice del processo di "apertura" della competizione politica. Se la prima alternanza del 1999 poteva apparire agli occhi di diversi analisti e commentatori come un incidente di percorso nella storia della sinistra bolognese, con le elezioni del 2016 si è reso evidente che non esiste più alcun reale vantaggio competitivo per i partiti eredi della tradizione comunista, se non quello derivante dalle divisioni e dalla carenza di leadership degli avversari. Tutte quelle condizioni che, per oltre cinquanta anni, avevano permesso la costruzione e il mantenimento di un "modello" politico fondato sul ruolo predominante (e a tratti egemone) di un partito di sinistra stanno, più o meno rapidamente, scomparendo. Nel corso degli ultimi venti anni, la politica bolognese ha progressivamente perso la sua eccezionalità, nel senso che è andata normalizzandosi rispetto alle tendenze già presenti in altri contesti locali cittadini. La città laboratorio per antonomasia, quella che doveva servire da vetrina del buongoverno per i partiti di sinistra, non esiste più. Ma, prima ancora delle conseguenze, restano da indagare nel dettaglio le cause che hanno reso Bologna una città come tante altre: politicamente contendibile ed elettoralmente incerta. Proprio di questo ci occuperemo nel resto dell'articolo.

\section{L'analisi dei flussi elettorali}

Quanto è "mobile", cioè disposto a cambiare di volta in volta le proprie preferenze, l'elettorato bolognese? Da dove provengono e verso dove si spostano gli elettori che hanno modificato il proprio voto? Quale percorso ha seguito il circa $40 \%$ di elettori che alle elezioni comunali del 2016 è approdato nell'astensionismo? Per cercare di capire come è cambiato negli ultimi 17 anni $^{1}$ il comportamento elettorale dei bolognesi, analizzeremo i flussi elettorali delle ultime cinque elezioni amministrative (1999, 2004, 2009, $2011 \mathrm{e}$ 2016) avvalendoci dei dati presentati nelle tabelle seguenti.

Abbiamo deciso di applicare il modello di Goodman (Goodman 1953; Schadee e Corbetta 1984) ai risultati elettorali dei candidati alla carica di sindaco. Questa scelta è perfettamente in linea con l'importanza che hanno via via assunto i candidati sindaco a partire dalla riforma del 1993 che ne introdusse l'elezione diretta. Da allora, nei comuni sopra i 15.000 abitanti, tutte le liste devono infatti essere collegate, come si sa, ad un candidato sindaco che non può essere sostituito da altro sindaco nel corso del proprio mandato elettorale a seguito di sfiducia consiliare o dimissioni senza che automaticamente si concluda anticipatamente la legislatura. Alla lista (o al gruppo di liste) collegata al sindaco eletto, tranne che in casi particolari ${ }^{2}$, viene attribuito almeno il $60 \%$ dei seggi

1 Abbiamo purtroppo dovuto escludere dall'analisi le elezioni del 1995 in quanto il Comune di Bologna ha effettuato un risezionamento tra tali elezioni e le successive che rende inapplicabile il metodo Goodman per l'analisi dei flussi. Per l'analisi dettagliata di quella competizione elettorale e della relativa mobilità, rimandiamo ai dati forniti da Baldini, Corbetta e Vassallo (2000).

2 Qualora il sindaco fosse eletto al primo turno, il premio scatta solo se anche le liste collegate abbiano ottenuto almeno il $40 \%$ dei voti. Inoltre, ma è un caso estremamente improbabile, se gli elettori assegnano la maggioranza assoluta dei voti ad una coalizione diversa da quella del sindaco vincitore, il consiglio viene composto in maniera proporzionale. 
consiliari. È il sindaco, infine, ad avere potere di nomina e revoca degli assessori. Tutti questi elementi fanno dei candidati sindaco gli attori principali della partita elettorale, e questo giustifica la scelta qui operata di effettuare l'analisi dei flussi sui voti ottenuti dai candidati alla carica di sindaco.

Le nostre analisi cominciano con i flussi tra primo e secondo turno del 1999 (tabella 2). Al ballottaggio del 1999, Guazzaloca, partito in svantaggio al primo turno, riesce ad ottenere la carica di sindaco principalmente grazie a due flussi importanti: in positivo, la maggioranza degli elettori dei candidati minori (altri) ha deciso di sostenere il candidato del centrodestra al ballottaggio; in negativo, Guazzaloca ha perso molti meno voti verso l'astensione rispetto a Bartolini $(1,1 \%$ degli elettori rispetto ad un'emorragia del 4,1\%). Nonostante quindi Bartolini sia riuscita a convincere un terzo circa dell'elettorato di Zamboni, la quota di voti persa verso l'astensione le è stata fatale, anche perché nessuno dei due candidati è riuscito a convincere a recarsi alle urne gli elettori che al primo turno si erano astenuti.

ТАв. 2 - Stime dei flussi elettorali tra il primo e il secondo turno delle elezioni comunali di Bologna (voto al sindaco) del 1999 (percentuali sul totale degli elettori).

\begin{tabular}{lcccccc}
\hline & Guazzaloca & Bartolini & Zamboni & Altri & Non voto & Totale \\
\hline Guazzaloca & 29,9 & & & 3,1 & & 33,6 \\
Bartolini & & 30,7 & 1,0 & & & 32,7 \\
Non voto & 1,1 & 4,1 & 2,4 & 2,2 & 23,9 & 33,7 \\
Totale & 31,0 & 34,8 & 3,4 & 5,4 & 25,4 & 100,0 \\
\hline
\end{tabular}

Fonte: Nostre elaborazioni su dati del sito web del Comune di Bologna.

Nota: Nella tabella sono riportati i flussi sul totale degli aventi diritto. Sono indicati solo i flussi superiori allo $0,5 \%$.

Il «non voto» comprende, oltre agli astenuti, anche le schede bianche e nulle. $\mathrm{Vr}=2,2$.

La tabella 3 mostra i flussi elettorali per l'elezione del sindaco di Bologna tra il 1999 e il2004. Appare da subito evidente come il cambiamento della legge elettorale abbia ridotto il numero di candidati rilevanti: da tre nel 1999 (erano quattro nel 1995) ad addirittura due solamente nel 2004, quando nessuno oltre a Guazzaloca (centrodestra) e Cofferati (centrosinistra) è riuscito a superare la soglia dello zero virgola. I due principali candidati alle elezioni del 2004 raccolgono il proprio consenso quasi esclusivamente all'interno del proprio bacino elettorale: nel polo di centrodestra, più di 9 elettori su 10 hanno confermato il proprio voto per Guazzaloca (pari al 28,3\% degli aventi diritto), così come nel polo del centrosinistra più di 9 elettori di Bartolini su 10 hanno sostenuto Cofferati nel 2004 (32,5\% degli aventi diritto). Solo piccole percentuali di elettori hanno deciso di cambiare schieramento tra il 1999 ed il 2004 passando da Guazzaloca a Cofferati $(2,7 \%)$ o, viceversa, da Bartolini a Guazzaloca $(1,4 \%)$. Gli elettori del candidato di sinistra, Zamboni, si sono riversati totalmente su Cofferati, che è stato anche in grado 
di recuperare, più di Guazzaloca, gli elettori che nel 1999 avevano deciso di votare per candidati minori $\left(2,1 \%\right.$ vs. $\left.1,7 \%^{3}\right)$ o di non recarsi alle urne $(3,9 \%$ vs $0,8 \%)$.

L'altro zoccolo duro dell'elettorato bolognese è rappresentato dagli astensionisti: il 19,8\% degli aventi diritto non è andato a votare né nel 1999 né nel 2004 (ciò significa che quasi 1'80\% di chi non aveva votato nel 1999 ha deciso di continuare a non votare nel 2004). Non si registrano invece perdite significative di elettori verso l'astensione da parte di nessuno dei principali candidati.

ТАВ. 3 - Stime dei flussi elettorali tra le elezioni comunali di Bologna (primo turno, voto al sindaco) del 1999 e del 2004 (percentuali sul totale degli elettori).

\begin{tabular}{lcccccc}
\hline & Guazzaloca & Bartolini & Zamboni & Altri & Non voto & Totale \\
\hline Cofferati & 2,7 & 32,5 & 3,1 & 2,1 & 3,9 & 44,3 \\
Guazzaloca & 28,3 & 1,4 & & 1,7 & 0,8 & 32,2 \\
Altri & & 0,8 & & 0,9 & 0,9 & 2,7 \\
Non voto & & & & 0,7 & 19,8 & 20,7 \\
Totale & 31,0 & 34,8 & 3,4 & 5,4 & 25,4 & 100,0 \\
\hline
\end{tabular}

Fonte: Nostre elaborazioni su dati del sito web del Comune di Bologna.

Nota: Nella tabella sono riportati i flussi sul totale degli aventi diritto. Sono indicati solo i flussi superiori allo $0,5 \%$. Il «non voto» comprende, oltre agli astenuti, anche le schede bianche e nulle. $\mathrm{Vr}=4,8$.

Anche il passaggio tra le elezioni del 2004 e quelle del 2009 è contrassegnato da una relativa stabilità inter e intra-coalizionale (vedi tabella 4). A turbarla soltanto la scelta del centrodestra di non sostenere l'ex sindaco Guazzaloca e di presentare al suo posto Alfredo Cazzola. Guazzaloca, infatti, ripresentandosi con una propria lista civica, è riuscito a trattenere 1' $8 \%$ degli elettori, pari ad un quarto di coloro che lo avevano votato nel 2004. I principali candidati, e cioè Delbono (centrosinistra) e Cazzola (centrodestra), hanno visto, con diversa intensità, confermata la fedeltà dell'elettorato del proprio schieramento: Delbono ha convinto più dell' $80 \%$ degli elettori di Cofferati (pari al 36,5\% degli aventi diritto), mentre Cazzola, nonostante abbia sofferto per la candidatura di Guazzaloca, è riuscito a trattenere più del $60 \%$ degli elettori di centrodestra (pari al 19,9\% degli aventi diritto). Gli scambi tra le due coalizioni sono stati minimi, con solo 1'1,2\% degli elettori che è passato dal centrodestra di Guazzaloca al centrosinistra di Delbono, così come, se si esclude il menzionato $8 \%$ di elettori che è rimasto fedele a Guazzaloca e non alla coalizione che lo sosteneva, anche i passaggi di elettori dalle due principali coalizioni del 2004 verso altri candidati (Guazzaloca, Favia, Monteventi ed altri) non ha superato 1'1,7\%. Gli elettori del centrosinistra di Cofferati hanno infatti cambiato idea solo in minima parte verso Guazzaloca (1,3\% del totale degli aventi diritto) e Favia (1,7\%), mentre gli elettori del centrodestra di Guazzaloca che hanno deciso di cambiare candidato o coalizione lo hanno fatto verso Delbono ( $1,2 \%$ degli aventi diritto) o verso i candidati minori $(1,5 \%)$.

3 È bene tuttavia notare anche che il centrosinistra abbia perso lo $0,8 \%$ del totale degli aventi diritto verso candidati minori, fenomeno non ravvisabile per il centrodestra. 
Le elezioni del 2009 sono anche quelle di esordio per il Movimento 5 Stelle. Il loro candidato, Giovanni Favia, ha raccolto i propri consensi quasi esclusivamente tra gli ex elettori di Cofferati e tra passati astensionisti. All'interno del bacino degli (ex) astensionisti, tuttavia, Cazzola è stato in grado di raccogliere più del triplo rispetto a Favia, pur trattandosi sempre di percentuali di elettori molto piccole.

Il popolo dell'astensione, infatti, si è dimostrato ancora una volta granitico. Il 16\% degli aventi diritto non si è recato alle urne né nel 2004 né nel 2009 (pari al 77\% degli astenuti del 2004). La maggior perdita verso l'astensione è stata subita dal centrosinistra: ben il 4,3\% degli elettori (e cioè poco meno di un decimo dell'elettorato di Cofferati) ha deciso di non sostenere Delbono non recandosi alle urne, mentre ha fatto lo stesso solo il $4,4 \%$ degli elettori di Guazzaloca, pari all' $1,4 \%$ degli aventi diritto.

TAB. 4 - Stime dei flussi elettorali tra le elezioni comunali di Bologna (primo turno, voto al sindaco) del 2004 e del 2009 (percentuali sul totale degli elettori).

\begin{tabular}{lccccc}
\hline & Cofferati & Guazzaloca & Altri & Non voto & Totale \\
\hline Delbono & 36,5 & 1,2 & & & 37,9 \\
Cazzola & & 19,9 & 0,9 & 1,6 & 22,3 \\
Guazzaloca & 1,3 & 8,0 & & & 9,7 \\
Favia & 1,7 & & 0,5 & 2,5 \\
Monteventi & & 1,5 & 1,0 & 1,2 \\
Altri & 4,3 & 1,4 & 1,5 & 1,1 & 3,0 \\
Non voto & 44,3 & 32,2 & 2,7 & 16,0 & 23,2 \\
Totale & & & 20,7 & 100,0 \\
\hline
\end{tabular}

Fonte: Nostre elaborazioni su dati del sito web del Comune di Bologna.

Nota: Nella tabella sono riportati i flussi sul totale degli aventi diritto. Sono indicati solo i flussi superiori allo $0,5 \%$. Il «non voto» comprende, oltre agli astenuti, anche le schede bianche e nulle. $\mathrm{Vr}=3,3$.

Partito da un notevole svantaggio al primo turno, Cazzola riesce solo a limitare la sconfitta raccogliendo più della metà degli elettori di Guazzaloca $(5,2 \%$ del corpo elettorale). Mentre, ancora una volta, i passaggi di schieramento sono nulli, Delbono vede confermato il voto di circa il $90 \%$ dei propri elettori del primo turno, perdendo un comunque non irrilevante 10\% verso l'astensione (vedi tabella 5). Anche Cazzola riesce a riportare alle urne gran parte del proprio elettorato, ma, in termini relativi, perde qualcosa in più verso l'astensione. L'elettorato di Guazzaloca, come accennato, si riversa per la maggioranza sul candidato maggiormente affine, e cioè Cazzola, preferendo poi in gran parte l'astensione al voto per Delbono. L'elettorato pentastellato e quello di Monteventi si dividono quasi equamente tra il sostegno a Delbono e l'astensione (dalla quale nessuno dei due candidati al ballottaggio riesce a recuperare voti). 
TАв. 5 - Stime dei flussi elettorali tra il primo e il secondo turno delle elezioni comunali di Bologna (voto al sindaco) del 2009 (percentuali sul totale degli elettori).

\begin{tabular}{lcccccccc}
\hline & Delbono & Cazzola & Guazzaloca & Favia & Monteventi & Altri & $\begin{array}{l}\text { Non } \\
\text { voto }\end{array}$ & Totale \\
\hline Delbono & 33,9 & & 1,3 & 1,2 & 0,6 & 1,1 & & 38,1 \\
Cazzola & & 18,8 & 5,2 & & & 0,6 & & 24,7 \\
Non voto & 4,0 & 3,6 & 3,2 & 1,3 & 0,6 & 1,3 & 23,2 & 37,2 \\
Totale & 37,9 & 22,3 & 9,7 & 2,5 & 1,2 & 3,0 & 23,2 & 100,0 \\
\hline
\end{tabular}

Fonte: Nostre elaborazioni su dati del sito web del Comune di Bologna.

Nota: Nella tabella sono riportati i flussi sul totale degli aventi diritto. Sono indicati solo i flussi superiori allo $0,5 \%$. Il «non voto» comprende, oltre agli astenuti, anche le schede bianche e nulle. $\mathrm{Vr}=3,4$.

Il passaggio tra il 2009 e il 2011 mette l'elettorato bolognese di fronte ad uno scenario nuovo, in cui nessuno schieramento ripresenta i medesimi candidato alla carica di sindaco. Ciononostante, gli elettori, soprattutto quelli del centrosinistra, sembrano mostrare ancora un elevato livello di fedeltà, preferendo il non-voto al cambio di schieramento. Così, come si nota nella tabella 6 , più dell' $85 \%$ dell'elettorato di Delbono accorda la sua fiducia a Merola (il 32,5\% degli aventi diritto), mentre solo quote marginali passano a candidati centristi $(0,6 \%$ del corpo elettorale) o all'astensione $(0,7 \%)$. Queste tuttavia sono anche le elezioni che rappresentano il passaggio all'età adulta per il Movimento 5 Stelle, che ottiene più della metà dei propri voti da elettori scontenti del centrosinistra. L'elettorato del centrodestra di Cazzola si dimostra meno fedele, preferendo il non-voto (4,1\% degli aventi diritto, pari a quasi il 19\% degli elettori di Cazzola) o i candidati centristi Aldrovandi e Corticelli (complessivamente il 2,4\% dell'intero elettorato) a Manes Bernardini. L'elettorato di Guazzaloca decide invece, per la maggior parte, di tornare nella casa del centrodestra, mentre i candidati centristi raccolgono un quarto delle preferenze accordate a Guazzaloca nel 2009 e Merola e Bugani si spartiscono equamente gli elettori restanti. Interessanti anche i casi di Favia e Monteventi. Il primo conserva più del $70 \%$ del proprio elettorato, perdendone però il $20 \%$ verso Merola, mentre il secondo cede tutto all'astensione. Astensione che si fa via via sempre più strutturale: infatti, quasi il $96 \%$ di chi non si era recato alle urne nel 2009 decide di non farlo nemmeno nel 2011, cosicché il bacino dell'astensione è composto quasi esclusivamente da elettori già astensionisti o da elettori di Cazzola che hanno deciso di non votare Bernardini. 
TAB. 6 - Stime dei flussi elettorali tra le elezioni comunali di Bologna (primo turno, voto al sindaco) del 2009 e del 2011 (percentuali sul totale degli elettori).

\begin{tabular}{|c|c|c|c|c|c|c|c|c|}
\hline & Delbono & Cazzola & Guazzaloca & Favia & Monteventi & Altri & $\begin{array}{l}\text { Non } \\
\text { voto }\end{array}$ & Totale \\
\hline Merola & 32,5 & & 0,7 & 0,5 & & & 0,7 & 35,1 \\
\hline Bernardini & & 15,4 & 5,6 & & & & & 21,1 \\
\hline Bugani & 3,7 & & 0,7 & 1,9 & & & & 6,6 \\
\hline Aldrovandi-Corticelli & 0,6 & 2,4 & 2,6 & & & & & 5,7 \\
\hline Altri & & & & & & & & 1,1 \\
\hline Non voto & 0,7 & 4,1 & & & 0,9 & 2,3 & 22,3 & 30,5 \\
\hline Totale & 37,9 & 22,3 & 9,7 & 2,5 & 1,2 & 3,0 & 23,2 & 100,0 \\
\hline
\end{tabular}

Fonte: Nostre elaborazioni su dati del sito web del Comune di Bologna.

Nota: Nella tabella sono riportati i flussi sul totale degli aventi diritto. Sono indicati solo i flussi superiori allo $0,5 \%$.

Il «non voto» comprende, oltre agli astenuti, anche le schede bianche e nulle. $\mathrm{Vr}=4,2$.

I flussi tra le elezioni del 2011 e quelle del 2016 raccontano invece tutta un'altra storia (vedi tabella 7). I tradizionali orientamenti di voto riescono sempre meno a fronteggiare le pressioni della destrutturazione. Pare evidente che il panorama politico sia irrimediabilmente mutato. Vediamo così che l'elettorato di Merola si suddivide in mille rivoli, e così quello degli altri candidati. Per quanto ancora più della metà degli elettori del centrosinistra continuino ad accordare la loro preferenza al medesimo schieramento, ben un quarto di loro (7,2\% del corpo elettorale) decide di non recarsi alle urne, mentre quasi il 14\% (48\% degli aventi diritto) degli elettori di Merola decide di passare a Bugani. Le restanti quote vengono all'incirca equamente spartite tra Borgonzoni $(1,2 \%)$, Bernardini $(1,2 \%)$ e altri $(1,4 \%)$. Il partito dell'astensione, infine, accoglie circa un quinto dell'elettorato di Merola (7,2\% degli aventi diritto).

Ancora una volta l'elettorato del centrodestra si dimostra meno fedele. Infatti, solo il 44\% di chi nel 2011 aveva votato Bernardini decide di confermare la propria preferenza per il centrodestra guidato, questa volta, da Borgonzoni e, come già avvenuto nel 2009 con Cazzola/Guazzaloca, una quota consistente (14,5\% circa dell'elettorato di Bernardini, pari al 3,1\% del corpo elettorale) di elettori rimane fedele al candidato e non allo schieramento. Più interessante, perché inconsueto, è invece il passaggio di una quota di elettori di pari entità (3\% degli aventi diritto) allo schieramento opposto capeggiato da Merola. L'astensione continua, infine, a rubare consensi al centrodestra: poco meno di un quarto (4,9\% del corpo elettorale) degli elettori di Bernardini decide di non recarsi alle urne.

Allo stesso modo, la quota più consistente degli elettori centristi (1,6\% degli aventi diritto) diserta le urne nel 2016, disperdendosi, per la quota restante, tra Borgonzoni $(0,8 \%)$, Bernardini (1,1\%), Martelloni $(0,8 \%)$ e altri $(0,7 \%)$. L'elettorato del Movimento 5 stelle sembra invece mostrare maggiori livelli di fedeltà: il $65 \%$ di chi aveva votato Bugani nel 2011 (pari al 4,3\% del corpo elettorale) riconferma la propria preferenza. Quote pari allo $0,7-0,8 \%$ degli elettori invece decidono di passare a Borgonzoni, Bernardini e Martelloni. 
Anche in questo caso, gli elettori meno mobili sono gli astensionisti: il 92,1\% di chi non si era recato alle urne nel 2011 (pari al 28,1\% del corpo elettorale) conferma la propria (non) scelta nel 2016. Queste fila vengono poi notevolmente ingrossate dagli elettori che passano da uno dei principali schieramenti (7,2\% dal centrosinistra e 4,9\% dal centrodestra) all'astensione.

ТАВ. 7 - Stime dei flussi elettorali tra le elezioni comunali di Bologna (primo turno, voto al sindaco) del 2011 e del 2016 (percentuali sul totale degli elettori).

\begin{tabular}{|c|c|c|c|c|c|c|c|}
\hline & Merola & Bernardini & $\begin{array}{l}\text { Corticelli- } \\
\text { Aldrovandi }\end{array}$ & Bugani & Altri & Non voto & Totale \\
\hline Merola & 19,3 & 3,0 & & & & & 22,9 \\
\hline Borgonzoni & 1,2 & 9,3 & 0,8 & 0,7 & & 0,7 & 12,9 \\
\hline Bugani & 4,8 & & & 4,3 & & & 9,6 \\
\hline Bernardini & 1,2 & 3,1 & 1,1 & 0,8 & & & 6,0 \\
\hline Martelloni & & 0,6 & 0,8 & 0,8 & & 1,4 & 4,1 \\
\hline Altri & 1,4 & & 0,7 & & & & 2,4 \\
\hline Non voto & 7,2 & 4,9 & 1,6 & & & 28,1 & 42,1 \\
\hline Totale & 35,1 & 21,1 & 5,7 & 6,6 & 1,1 & 30,5 & 100,0 \\
\hline
\end{tabular}

Fonte: Nostre elaborazioni su dati del sito web del Comune di Bologna.

Nota: Nella tabella sono riportati i flussi sul totale degli aventi diritto. Sono indicati solo i flussi superiori allo $0,5 \%$.

Il «non voto» comprende, oltre agli astenuti, anche le schede bianche e nulle. $\mathrm{Vr}=5,5$.

Nonostante i numerosi elementi di incertezza, il ballottaggio ha confermato il vincitore del primo turno, rendendo possibile così il secondo mandato per il sindaco Merola. Se osserviamo i flussi elettorali avvenuti tra i due turni, la vittoria del centrosinistra è frutto sostanzialmente di due fattori. Da un lato, la tenuta del suo elettorato del primo turno: il 20,4\% degli elettori bolognesi avevano votato Merola il 5 giugno e hanno riconfermato il voto due settimane dopo. Dall'altro lato, il PD e i suoi alleati riescono ad allargare la propria platea elettorale grazie al decisivo contributo degli elettori di Martelloni (3,5\% dell'intero elettorato passa da Martelloni a Merola) e dei Verdi (altri), i quali si erano formalmente «apparentati» col centrosinistra.

Anche se a Borgonzoni non è riuscita la rimonta, la crescita dei suoi consensi nel ballottaggio è stata soprattutto il prodotto degli elettori che, al primo turno, avevano sostenuto Bernardini o Bugani (vedi tabella 8). Se per l'elettorato del «civico» Bernardini era naturale la convergenza verso la candidata del centrodestra, il comportamento degli elettori pentastellati era meno prevedibile. L'elettorato grillino si è infatti sostanzialmente spaccato a metà: una parte (il 4,4\% degli aventi diritto) ha scelto l'astensione, mentre l'altra parte (il 4,1\%) ha optato per un voto alla candidata di un centrodestra a trazione leghista. Solo una quota minoritaria dell'elettorato di Bugani (cioè, 1'11,5\%) ha preferito votare Merola piuttosto che non recarsi alle urne o convergere su Borgonzoni. 
ТАв. 8 - Stime dei flussi elettorali tra il primo e il secondo turno delle elezioni comunali di Bologna (voto al sindaco) del 2016 (percentuali sul totale degli elettori).

\begin{tabular}{lcccccccc}
\hline & Merola & Borgonzoni & Bugani & Bernardini & Martelloni & Altri & $\begin{array}{c}\text { Non } \\
\text { voto }\end{array}$ & Totale \\
\hline Merola & 20,4 & & 1,1 & 0,7 & 3,5 & 2,0 & & 27,9 \\
Borgonzoni & & 12,9 & 4,1 & 5,3 & & & 0,9 & 23,2 \\
Non voto & 2,4 & & 4,4 & & 0,5 & & 41,2 & 49,0 \\
Totale & 22,9 & 12,9 & 9,6 & 6,0 & 4,1 & 2,4 & 42,1 & 100,0 \\
\hline
\end{tabular}

Fonte: Nostre elaborazioni su dati del sito web del Comune di Bologna.

Nota: Nella tabella sono riportati i flussi sul totale degli aventi diritto. Sono indicati solo i flussi superiori allo $0,5 \%$. Il «non voto» comprende, oltre agli astenuti, anche le schede bianche e nulle. $\mathrm{Vr}=2,7$.

Ai fini della comparazione sincronica tra i casi analizzati in questo numero monografico dei Quaderni e diacronica tra i flussi di una medesima città, utilizziamo la stessa misura sintetica proposta da Vignati nel saggio su Milano e ripresa anche da Fruncillo e Gentilini nel saggio su Napoli, suddividendo il corpo elettorale in sei categorie. La classificazione non sfugge a qualche elemento di arbitrarietà, soprattutto nei casi in cui l'offerta politica si ristruttura in maniera così ampia da rendere complicato distinguere gli elettori "stabili" da quelli “mobili". Nel caso bolognese ciò avviene soprattutto all'interno del centrodestra, dove Guazzaloca prima e Bernardini poi hanno deciso di ripresentarsi agli elettori con proprie liste civiche, mentre la propria coalizione gli preferiva un altro candidato. In casi come questi, gli elettori sono stati classificati come "mobili", facendo prevalere la lealtà alla forza politica piuttosto che alla figura del candidato.

La prima categoria fa riferimento agli elettori "stabili", e cioè a tutti quegli elettori che decidono di confermare il proprio voto tra un'elezione e l'altra. Racchiude principalmente due tipi di elettori: i fedeli del centrosinistra e i fedeli del centrodestra. Oltre a questi, recentemente vi si sono aggiunti gli elettori fedeli al Movimento 5 Stelle. La seconda categoria comprende gli "smobilitati" e i "rimobilitati", e cioè tutti quegli elettori che passano, nel primo caso, dal voto al non-voto, mentre, nel secondo caso, percorrono la strada inversa, tornando a recarsi alle urne dopo averle disertate nella tornata precedente. La terza e la quarta categoria fanno riferimento agli elettori "mobili". Sono stati individuati due tipi di elettori mobili: quelli che abbiamo chiamato "convertiti", che si muovono dal centrosinistra al centrodestra e viceversa, e quelli che abbiamo chiamato "mobili periferici", che passano dal candidato di una delle due principali coalizioni ad altri candidati (o viceversa) oppure che votano, in entrambe le elezioni, per candidati di "terze forze". La quinta categoria comprende gli elettori che definiamo come "trasportati". Costoro in realtà non si muovono per volontà propria, ma per quella del proprio partito che decide di non presentarsi con un proprio candidato, ma di sostenere il candidato di una delle due principali coalizioni. Ciò è avvenuto a Bologna una sola volta, tra le elezioni del $1999 \mathrm{e}$ quelle del 2004, quando gli elettori di Zamboni (Rifondazione comunista), furono "trasportati" all'interno del blocco di centrosinistra che sosteneva Cofferati. Infine, la sesta e ultima categoria riguarda gli "assenti", e cioè gli astensionisti cronici. 
ТАВ. 9 - Classificazione dell'elettorato bolognese in relazione alla stabilità/mobilità del voto nelle elezioni comunali.

\begin{tabular}{lcccc}
\hline & $1999-2004$ & $2004-2009$ & $2009-2011$ & $2011-16$ \\
\hline Stabili & 60,8 & 56,4 & 52,3 & 32,8 \\
Smob./rimob. & 6,4 & 11,9 & 9,2 & 16,4 \\
Convertiti & 4,1 & 1,2 & & 4,3 \\
Mobili periferici & 5,7 & 14,5 & 16,2 & 18,4 \\
Trasportati & 3,1 & & & \\
Assenti & 19,8 & 16,0 & 22,3 & 28,1 \\
Totale & 100,0 & 100,0 & 100,0 & 100,0 \\
\hline
\end{tabular}

Fonte: Nostre elaborazioni su dati tratti dal sito del comune di Bologna.

La tabella 9 riporta i dati relativi alla stabilità/mobilità elettorale dei bolognesi. Salta subito all'occhio la netta dominanza, in tutte e quattro le occasioni, della quota di elettori stabili. Tuttavia, tale quota è andata via via riducendosi passando dall' $60,8 \%$ tra il 1999 e il 2004 al 32,8\% tra il 2011 e il 2016. Al contempo, è invece aumentata la quota di elettori che non si recano alle urne (assenti): dal 19,8\% del 1999-2004 al 28,1\% del 2011-2016. La diminuzione degli stabili unita ad un aumento degli esclusi ci porta a concludere che, in realtà, la riduzione nel numero di coloro che restano fedeli al proprio schieramento è via via sempre maggiore. Ciò non deve tuttavia sorprenderci in quanto è ormai da decenni in corso un processo di indebolimento delle (sub)culture politiche (e del voto di appartenenza che ne consegue) a favore di un voto sempre più mobile unito ad una forte destrutturazione del sistema partitico (Sani 1992; Chiaramonte e Emanuele 2014; Pasquino e Valbruzzi 2015).

I livelli di mobilitazione/smobilitazione sono altalenanti, ma tendenzialmente in crescita, mentre i passaggi tra le due principali coalizioni ("convertiti") rappresentano una quota molto bassa dell'elettorato (toccano l'apice nel passaggio 2011-2016 con il $4,3 \%$ ). Molto più alta ed in decisa e continua crescita risulta invece la percentuale dei "mobili periferici", e cioè di coloro che si muovono da/verso "terze forze": dal 5,7\% del 1999-2004 al 18,4\% del 2011-2016. La riduzione dell'elettorato stabile, insieme all'aumento dei mobili periferici, ci porta a concludere che è in atto un vero e proprio scardinamento del bipolarismo che pare tenere, in molti casi, solo grazie agli effetti della legge elettorale, ma che, in molti altri, è già spezzato dall'avanzamento di terze forze come il Movimento 5 Stelle. 


\section{Bipolarismo, frammentazione e personalizzazione}

Anche se rimane una tradizione di comportamenti, elettorali e non, che tende strutturalmente a privilegiare i partiti del centrosinistra, nel corso degli ultimi anni e, in particolare nel 2016, abbiamo potuto assistere ad un progressivo scongelamento del voto di appartenenza e ad una maggiore disponibilità a muoversi lungo l'intero spettro dell'offerta politica. Nel paragrafo precedente ci siamo concentrati prevalentemente sul lato della domanda (o della risposta) elettorale, cercando di mettere in luce le trasformazioni negli orientamenti e negli atteggiamenti di voto dei cittadini bolognesi. Ora, invece, intendiamo spostare la nostra attenzione sul lato della proposta politica, vale a dire delle diverse opzioni di voto che i partiti hanno sottoposto al giudizio degli elettori. Anche in questo caso, per molto tempo, sicuramente fino al crollo del Muro di Berlino e in presenza in Italia di un sistema partitico consolidato e sufficientemente strutturato, la volatilità sul piano dell'offerta partitica nel contesto bolognese è stata decisamente ridotta. Se a livello nazionale si consolidava quella sorta di «bipolarismo imperfetto» descritto da Giorgio Galli (1967), che prevedeva il governo incontrastato del polo democristiano su quello comunista, nel contesto bolognese la situazione si presentava completamente invertita: era il "polo" comunista, fortemente radicato e strutturato sul territorio e nella società, che rendeva praticamente impossibile l'avverarsi dell'alternanza. L' "imperfezione" del bipolarismo bolognese era il prodotto, da un lato, dalla forza elettorale ed organizzativa del PCI e, dall'altro lato, dall'incapacità dei partiti all'opposizione di formulare e coordinare una proposta politica alternativa rispetto a quella delle forze di sinistra.

All'intero di questo scenario, le elezioni bolognesi del 1995 - le prime con l'elezione diretta del sindaco - rappresentano il momento conclusivo di un sistema partitico che, fino ad allora, si era caratterizzato per l'assenza di una reale competizione per il governo della città e un livello piuttosto elevato di frammentazione. In termini sartoriani (1976), con il voto del 1995 si chiude a Bologna la fase di un sistema a partito predominante e incomincia lentamente a vedere la luce il tentativo di costruire un formato bipolare, composto da due coalizioni disposte ad alternarsi al potere. In maniera meno lineare rispetto a quello che si stava affermando a livello nazionale, anche il sistema partitico bolognese aveva intrapreso la strada - grazie agli incentivi inseriti all'interno del nuovo sistema elettorale - del bipolarismo (senza più "imperfezioni”, almeno in chiave teorica). Questa trasformazione è ben visibile nei dati raccolti nella tabella 10, che riporta i valori di alcuni indici di concentrazione elettorale sui due principali partiti o schieramenti/poli. L'indice di bipolarismo, ovvero la somma dei voti raccolti dalle due coalizioni che hanno ottenuto il maggior numero di consensi, mostra chiaramente il rafforzamento della struttura bipolare all'interno del sistema politico bolognese. Nel 1995 l'indice di bipolarismo era pari a $68,1 \%$, mentre negli anni successivi è quasi sempre superiore all' $80 \%$, raggiungendo il massimo nel 2004 con il 96\%. L'unica eccezione è rappresentata dalle elezioni del 2016, quando il bipolarismo si riduce drasticamente al 63,8\% dei voti. Questa è, però, una eccezione poco eccezionale e che, infatti, si inserisce perfettamente nel nuovo quadro nazionale, tendenzialmente tripolare, che si è aperto con le elezioni politiche del 2013. 
ТАВ. 10 - Valori degli indici di bipolarismo, bipartitismo e frammentazione partitica registrati nelle elezioni comunali di Bologna, 1995-2016.

\begin{tabular}{cccccccc}
\hline & $\begin{array}{c}\text { Bipolarismo } \\
(\%)\end{array}$ & $\begin{array}{c}\text { Bipartitismo } \\
(\%)\end{array}$ & N. liste & Neff voti & $\begin{array}{c}\text { Neff } \\
\text { seggi }\end{array}$ & $\begin{array}{c}\text { Disproporzionalità } \\
(\%)\end{array}$ & $\begin{array}{c}\text { Margine di } \\
\text { vittoria (\%) }\end{array}$ \\
\hline 1995 & 68,1 & 56,1 & 13 & 4,5 & 3,1 & 10,8 & 32,3 \\
1999 & 87,4 & 41,0 & 16 & 7,4 & 5,3 & 10,5 & 5,1 \\
2004 & 96,0 & 54,9 & 18 & 5,1 & 3,9 & 5,9 & 15,3 \\
2009 & 79,4 & 55,4 & 23 & 4,7 & 2,8 & 11,9 & 20,3 \\
2011 & 81,6 & 53,5 & 17 & 4,8 & 3,1 & 10,7 & 20,1 \\
2016 & 63,8 & 52,1 & 17 & 5,4 & 2,2 & 22,9 & 17,2 \\
\hline
\end{tabular}

Note: Nostra elaborazione sui dati del Ministero dell'Interno.

Osservata in questa prospettiva, l'evoluzione del sistema partitico bolognese appare combaciare, per molti aspetti, con quella sperimentata a livello nazionale. Sembrano essere due, in particolare, le somiglianze tra i due livelli, con una sola, grande differenza. La prima somiglianza riguarda la tempistica con cui anche la città di Bologna si è adeguata e ha seguito l'avvento del bipolarismo in Italia. Anche se con qualche ritardo, il bipolarismo coalizionale si è progressivamente inserito nella vita politica bolognese e ha incominciato a influenzare tanto l'offerta partitica quanto la domanda elettorale. Ugualmente, nel momento in cui quel formato di competizione inter-partitica è entrato in crisi nel 2013, principalmente per l'ingresso di un terzo attore "rilevante" in termini sistemici come il Movimento 5 stelle, anche sotto le Due Torri si è incominciata a incrinare la forza del bipolarismo municipale.

La seconda somiglianza tra i due sistemi partitici (locale e nazionale) riguarda il tipo di bipolarismo che si è effettivamente affermato nel nostro paese. Anche se su questo aspetto le definizioni abbondano e le analisi non sempre convergono, non c'è dubbio che il bipolarismo italiano, sia nazionale che municipale, si sia caratterizzato per la sua estrema frammentazione. Come mostrano i dati della tabella 10 in riferimento al «numero effettivo dei partiti» (Neff in voti e in seggi) (Laakso e Taagepera 1979) e al numero di liste presentate in ogni elezioni, il formato del sistema partitico è rimasto altamente frammentato tra una pluralità di liste e partiti. Il numero effettivo di partiti, calcolato in relazione ai seggi in consiglio comunale conquistati da ciascuna lista, tocca il livello massimo nel 1999 (5,3), l'anno in cui avvenne la prima alternanza al governo della città di Bologna, mentre per ciò che riguarda il numero di liste presentate l'apice della frammentazione si raggiunge nel 2009, quando l'elettore si trova a dover/poter scegliere tra 23 liste. È assolutamente interessante notare, in questo caso, che il valore più basso registrato dal numero effettivo di partiti in seggi si raggiunge nel $2016(2,2)$, e cioè in coincidenza con il ritorno del multipolarismo nelle vesti di una struttura tripolare. Questo dato, che sembrerebbe andare in controtendenza rispetto al quadro nazionale, si spiega con la forza ancora fortemente riduttiva/distorsiva del sistema elettorale utilizzato a livello comunale. Per misurare la forza di questo effetto, nella tabella 10 abbiamo riportato 
anche i valori dell'indice di disproporzionalità (Gallagher 1991), allo scopo di misurare l'effetto distorcente esercitato da un determinato sistema elettorale nel passaggio dai voti ottenuti ai seggi conquistati. Come si nota chiaramente, la disproporzionalità delle elezioni raggiunge il valore più elevato $(22,2)$ proprio nelle elezioni comunali del 2016. Questo significa che l'effetto manipolativo del sistema elettorale sulla struttura partitica ha avuto un impatto decisivo (e fortemente disproporzionale) nella ripartizione dei seggi in consiglio e, quindi, sui rapporti di forza tra i partiti. In pratica, nel momento in cui l'elettorato bolognese - in linea con quello italiano - tendeva a suddividersi in tre blocchi, i meccanismi istituzionali ed elettorali hanno forzato il tripolarismo all'interno dello schema bipolare, producendo così il massimo grado di distorsione o disproporzionalità nel passaggio dell'arena elettorale a quella partitica.

L'unica differenza di rilievo tra il livello municipale bolognese e quello nazionale ha riguardato la forza o, meglio, la compattezza del polo di centrodestra. Mentre sul piano nazionale, grazie anche alla leadership senza rivali di Silvio Berlusconi, lo schieramento di centrodestra si è presentato quasi sempre unito o, comunque, in grado di competere seriamente con la coalizione di centrosinistra, a Bologna il centrodestra ha affrontato le elezioni spesso in maniera divisa e con più di un candidato. Nei due casi in cui l'intero centrodestra ha sostenuto una sola candidatura, per di più (e significativamente) di natura civica, la sua capacità di sfidare lo schieramento opposto è cresciuta notevolmente, portando in entrambi i casi gli avversari alla sfida del ballottaggio (1999 e 2009). Tuttavia, la natura composita e frazionata del centrodestra bolognese ha rappresentato un limite al consolidamento di una struttura interpartitica completamente bipolare, facendo dell'alternanza più un'ipotesi teorica che una realtà empirica concretamente realizzabile. La scarsa competitività del centrodestra bolognese emerge anche dall'analisi dei margini di vittoria (in termini di voti percentuali) tra le due coalizioni principali che si sono confrontate alle elezioni. Anche in questo caso, non è una sorpresa che la maggiore competitività o incertezza dell'esito elettorale si sia riscontrato nel 1999 e nel 2004, ossia nelle uniche due elezioni che hanno prodotto un'alternanza al potere: nel primo caso, dal centrosinistra al centrodestra a trazione civica e, nel secondo caso, nella direzione opposta, con l'amministrazione di Sergio Cofferati.

Nell'insieme, l'analisi condotta fin qui sulle trasformazioni nella struttura del sistema partitico ha rivelato sia uniformità che difformità rispetto all'evoluzione osservata a livello nazionale. Anche a Bologna, seppur più faticosamente, nel corso degli ultimi venti anni si è instaurata una forma di bipolarismo frammentato, spesso litigioso e disomogeneo al proprio interno. Tuttavia, rispetto al quadro nazionale, la situazione politica di Bologna risulta ancora in parte condizionata dall'esistenza di una tradizione di cultura politica che tende a premiare, abbastanza stabilmente e strutturalmente, lo schieramento di centrosinistra, anche per ritardi ed errori strategici dei partiti avversari. Ciò nonostante, questa sorta di vantaggio competitivo per i partiti eredi della forza organizzativa del PCI sembra ormai entrata in una fase discendente, rendendo molto più incerti e imprevedibili i comportamenti degli elettori e, di riflesso, i risultati delle elezioni.

Finora ci siamo limitati ad analizzare le caratteristiche del sistema partitico bolognese per quel che concerne i cambiamenti nei rapporti di forza e nelle relazioni tra $i$ 
singoli partiti o le coalizioni. Non è stato detto ancora nulla in merito ai rapporti che si sono venuti a creare tra $\mathrm{i}$ "nuovi" sindaci demo-eletti e le loro rispettive organizzazione partitiche. Come è stato detto nel secondo paragrafo, Bologna è stata per lungo tempo un esempio classico di governo di partito, all'interno del quale la rete organizzativa del partito tendeva a prevalere su quella dell'esecutivo e del suo esponente temporaneo. Con l'introduzione dell'elezione diretta del sindaco, sebbene con qualche ritardo dovuto all'esistenza di un passato difficile da superare, anche a Bologna si è fatta lentamente strada la personalizzazione della politica, in tutte le sue varianti e sfumature. Naturalmente, la prima espressione della personalizzazione è quella che riguarda il vertice dell'esecutivo, reso il vero dominus nell'architettura istituzionale complessiva del comune. La demoelezione implica, ovviamente, che tutti i fari principali della campagna elettorale sono puntati sui candidati sindaci e, di conseguenza, diventa importante, se non prioritario, individuare quelle candidature in grado di attrarre il voto di una platea vasta di elettori, possibilmente superiore rispetto a quella dei partiti a loro sostegno. In questo rapporto uno-a-molti tra il candidato leader e gli elettori diventa fondamentale individuare una personalità che riesca ad elevarsi - in termini elettorali, e non solo - al di sopra della propria coalizione o del proprio partito di riferimento.

Per poter cogliere questo aspetto della personalizzazione, abbiamo calcolato la percentuale di voti ai soli candidati sindaci rispetto al totale dei voti della coalizione (tasso di personalizzazione). Questo rapporto ci permette di valutare quanto una determinata competizione elettorale sia guidata $\mathrm{o}$, meglio, incentrata sulla figura dei leader oppure fondata sulle differenze tra i partiti. Come mostra la tabella 11, i candidati hanno sempre apportato un contributo elettorale positivo rispetto alla loro coalizione e ciò significa che la scelta del candidato ha assunto un'importanza cruciale per l'esito della competizione. A tal riguardo, è interessante notare che la macro-personalizzazione elettorale raggiunge il suo livello massimo proprio in occasione delle elezioni amministrative del 2004, quando entrambi i principali poli schierano candidati sindaci forti, con una storia personale non riconducibile esclusivamente al perimetro dei partiti a loro sostegno. Sia Cofferati che Cazzola riescono, infatti, ad attrare una quota significativa di voti personali che difficilmente sarebbero stati intercettati dalle organizzazioni di partito.

ТАВ. 11 - Tassi di personalizzazione e tassi di preferenza nelle elezioni comunali di Bologna, 1995-2016 (valori percentuali).

\begin{tabular}{lcc}
\hline & Macro-personalizzazione (\%) & Micro-personalizzazione (\%) \\
1995 & 7,0 & - \\
1999 & 10,3 & 21,4 \\
2004 & 12,5 & 29,1 \\
2009 & 5,9 & 27,8 \\
2011 & 10,1 & 36,8 \\
2016 & 2,7 & $18,4 *$ \\
\hline
\end{tabular}

Note: * Elezioni con doppia preferenza di genere, il denominatore è quindi moltiplicato per 2. 
In chiave diacronica, è possibile anche individuare un tendenziale aumento della macro-personalizzazione elettorale (con l'eccezione delle elezioni del 2009), che però sembra subire una netta battuta d'arresto nel 2016, quando il contributo dei singoli candidaci sindaci si riduce, in media, ad appena il 2,7\%, il valore più basso tra tutti quelli registrati nelle sei elezioni bolognesi qui esaminati. Per quanto possano esistere interpretazioni divergenti di questo dato in controtendenza, la spiegazione più convincente sembra rimandare alla questione, già esaminata in precedenza, del tripolarismo: con un'offerta partitica più ricca $\mathrm{o}$, comunque, strutturata su tre poli rivali, una buona parte di elettori che in passato avevano espresso un voto al solo candidato sindaco potrebbero aver trovato convincenti le proposte di uno dei partiti in lizza. Un'altra spiegazione potrebbe essere legata, invece, ad una considerazione storica dell'ultimo ventennio e, nello specifico, al ruolo che i sindaci hanno assunto nel corso di questo periodo. Con l'introduzione della demo-elezione nei primi anni Novanta del secolo scorso, i sindaci hanno assunto una posizione di assoluta rilevanza sia rispetto ai partiti che di fronte all'opinione pubblica. L'espressione "stagione dei sindaci" serviva appunto a descrivere quella fase in cui sembrava esistere un rapporto privilegiato o quasi una luna di miele prolungata tra gli elettori e i loro primi cittadini. Tuttavia, per una pluralità di ragioni che rimandano principalmente alle crescenti difficoltà economiche degli enti locali e ai sempre più ristretti margini di manovra degli esecutivi municipali, anche la stagione dei sindaci appare una pagina chiusa della storia politica italiana. Di conseguenza, il dato relativo alla macropersonalizzazione del 2016 potrebbe riflettere questa tendenza di lungo periodo e segnalare che anche a Bologna quella stagione (mai, peraltro, particolarmente calda o forte) è oramai soltanto un ricordo.

Passando ad osservare la distribuzione della macro-personalizzazione tra i principali schieramenti politici (tabella 12), ci sono diversi aspetti che meritano un approfondimento. In primo luogo, a parte alcuni episodi eccezionali, i candidati di centrosinistra mostrano una minore capacità di attrarre consensi personali rispetto a quelli di centro (come Guazzaloca) o di centrodestra (Berselli, Cazzola, Bernardini). In tal caso, la variabile che interviene in questo rapporto è collegata alla maggiore presenza organizzativa dei partiti di sinistra, in grado di orientare più efficacemente il comportamento dei propri elettori. Inoltre, un basso livello di macro-personalizzazione nell'ambito del centrosinistra può essere ricondotto anche ad una particolare tradizione culturale che ha fatto sempre prevalere l'apparato partitico sulle singole personalità. Come hanno giustamente sottolineato Baldini, Corbetta e Vassallo (2000, p. 238), le leadership politiche nel contesto dei partiti (post)comunisti bolognesi sono spesso state «un prodotto dell'ufficio piuttosto che di straordinarie qualità comunicative». In pratica, la carriera all'intero del partito finiva per prevalere sulle doti personali dei candidati/eletti. Questa tradizione ha avuto inevitabili conseguenze nella fase in cui la leadership del sindaco ha assunto un ruolo fondamentale a seguito della riforma della legge elettorale e della forma di governo municipale. Da questo punto di vista, la decisione del centrosinistra di candidare Sergio Cofferati nel 2004, accettando un candidato estraneo alla tradizione bolognese, attesta chiaramente le difficoltà ad affrontare una competizione elettorale centrata sulla figura dei candidati sindaci. 
ТАВ. 12 -Tassi di personalizzazione nelle elezioni comunali di Bologna, 1995-2016, distinti per area politica.

\begin{tabular}{lcccccc}
\hline & Sinistra & Centrosinistra & Centro & Centrodestra & Lega & M5s \\
\hline 1995 & $-1,3$ & 7,0 & 8,0 & 7,4 & $-1,7$ & \\
1999 & 2,1 & 10,5 & & 11,5 & 2,6 & \\
2004 & & 10,1 & & 17,0 & $-9,9$ & \\
2009 & 1,6 & 3,8 & 9,3 & 6,4 & & 13,2 \\
2011 & 29,7 & 3,3 & 17,1 & 19,1 & & 11,0 \\
2016 & 3,3 & 2,9 & 7,4 & 4,0 & & 2,7 \\
\hline
\end{tabular}

Nell'ambito del centrodestra, il problema sembra di natura totalmente opposta: esistono candidati che possono godere di un discreto seguito personale, ma hanno spesso l'appoggio di partiti deboli o non in grado di incanalare le preferenze del loro elettorato potenziale. Questo è il motivo per cui i candidati di centrodestra, così come quelli di centro, mostrano un livello di macro-personalizzazione maggiore rispetto ai loro rivali di centrosinistra. In un certo senso, la forza dei candidati sindaco scelti dai partiti di centro o di destra sono il riflesso, o forse la conseguenza, della debolezza organizzativa degli apparati partitici che dovrebbero sorreggerli. In assenza di tali strutture radicate sul territorio cittadino, i partiti di centrodestra hanno preferito concentrare la loro attenzione e le loro fatiche nella ricerca di candidati forti, con una storia personale in grado di superare i recinti elettorali dei singoli partiti.

È importante segnalare, inoltre, il dato sulla macro-personalizzazione del Movimento 5 Stelle, che ha preso parte alle ultime tre elezioni amministrative. In questo caso, appare piuttosto evidente il trend declinante della personalizzazione all'interno del "partito di Grillo". Nel 2009, quando il candidato pentastellato era il giovane Giovanni Favia (poi espulso dal Movimento), la macro-personalizzazione raggiunge il valore massimo di 13,2, mentre scende a 11,0 nel 2011 e a 2,7 nel 2016 con la candidatura di un esponente molto vicino a Grillo e al cosiddetto direttorio come Massimo Bugani. Nelle ultime due elezioni, la scelta di un candidato perfettamente allineato alla strategia, sia nazionale che locale, del partito pentastellato ha comportato una minore capacità di attrazione nei confronti di quegli elettori interessati a esprimere unicamente una preferenza personale e slegata da ogni riferimento partitico.

Infine, per quanto riguarda la macro-personalizzazione delle liste minori, tra cui rientrano quelle dei partiti di estrema sinistra o della Lega Nord, è evidente una tendenziale debolezza in termini di leadership. In molte elezioni, infatti, le liste di questi partiti hanno ottenuto più voti rispetto a quelli raccolti dai soli candidati sindaci: un dato che può essere spiegato facendo riferimento alla diversa propensione degli elettori dei partiti più piccoli a ricorrere al voto disgiunto, spezzando il proprio sostegno tra una lista di partito e il candidato sindaco di un altro schieramento. Questo comportamento strategico spiega il basso livello di macro-personalizzazione per i partiti minori.

Ciò detto, il fenomeno della personalizzazione non si esaurisce nel rapporto che 
lega verticalmente un leader ai propri elettori. La seconda faccia della personalizzazione, solitamente definita come micro-personalizzazione, è il prodotto di una rete orizzontale di rapporti personali uno-a-uno che serve a creare un contatto diretto tra l'elettore e il candidato. Come notato da Bolgherini e Musella (2006, p. 224), questo rapporto micropersonale «si instaura su piccola scala, in modo diretto e non mediato né dalla distanza né dai grandi numeri, e si sviluppa sul territorio, su un'area ben definita in cui i fattori di conoscenza personale diventano essenziali».

Nel caso specifico, con il concetto di «micro-personalizzazione» ci riferiamo alla competizione tra $\mathrm{i}$ candidati in lista per la carica di consigliere comunale e quindi analizziamo il voto di preferenza. Misuriamo la micro-personalizzazione attraverso il tasso di preferenza, ossia come il rapporto tra voti di preferenza espressi e voti di preferenza esprimibili ${ }^{4}$ (il denominatore è dato dai voti validi ai partiti).

In generale, i dati presentati in tabella 11 mostrano un incremento del tasso di preferenza dal 21,4 del 1999 al 36,8 del 2011. Questa tendenza però muta radicalmente nel 2016, quando il tasso si dimezza scendendo al 18,4. Queste elezioni sono state anche le prime (e le uniche svolte sino ad ora) con la doppia preferenza di genere, un meccanismo che permette agli elettori di esprimere due voti di preferenza per i candidati alla carica di consigliere comunale a patto che il secondo sia espresso per un candidato di genere diverso dal primo. Se quindi, da un lato, gli elettori hanno mostrato un interesse crescente per la carica di consigliere comunale, dall'altro il meccanismo della doppia preferenza di genere sembra non aver preso piede. Non abbiamo modo di sapere con certezza se gli elettori che hanno espresso preferenze nel 2016 ne abbiano espresse una ciascuno oppure due. Nel primo caso, potremmo concludere che la percentuale di elettori che ha espresso preferenze è rimasta esattamente identica a quella del 2011. Nel secondo caso, che si è dimezzata. Nel caso più probabile, il dato reale si trova nel mezzo, ma, essendo il tasso dato dal rapporto tra voti di preferenza espressi e voti di preferenza esprimibili, dobbiamo limitarci a rilevare che, raddoppiate le possibilità di scelta, gli elettori bolognesi non ne hanno fatto maggiore uso che in passato. Sappiamo che il processo di apprendimento elettorale richiede almeno due o tre tornate elettorali e quindi ci vorrà almeno un decennio per poter valutare compiutamente gli esiti sulla micro-personalizzazione dell'introduzione della doppia preferenza di genere.

Oltre alla micro-personalizzazione a livello complessivo, è interessante analizzare anche le differenze tra forze politiche. A questo scopo, sono stati calcolati i tassi di micropersonalizzazione per i diversi schieramenti: sinistra (lista/e collegate a Zamboni nel 1999 e Martelloni nel 2016), centrosinistra (lista/e collegate a Bartolini nel 1999, Cofferati nel 2004, Delbono nel 2009 e Merola nel 2011 e nel 2016), centro (lista/e collegate a Guazzaloca nel 2009 e Corticelli e Aldrovandi nel 2011), centrodestra (lista/e collegate a Guazzaloca nel 1999 e nel 2004, Cazzola nel 2009, Bernardini nel 2011 e Borgonzoni nel 2016) e Movimento 5 stelle (lista collegata a Favia nel 2009 e Bugani nel 2011 e nel 2016).

4 Dato che le elezioni del 2016 sono state le uniche a svolgersi consentendo all'elettore di esprimere una doppia preferenza di genere, in questo caso il denominatore sarà dato dai voti validi ai partiti moltiplicati per due. 
I dati presentati in tabella 13 mostrano che, in generale, la coalizione di centrosinistra ha (quasi) sempre avuto livelli di micro-personalizzazione superiori agli altri schieramenti. Ed è anche la forza politica, se si esclude la sinistra presente nella nostra analisi solo nel 1999 e nel 2016, che ha visto i propri tassi di preferenza incrementare maggiormente fino ad arrivare a valori più che doppi. Anche l'elettorato del centrodestra ha vieppiù aumentato la propria propensione a esprimere preferenze per i candidati alla carica di consigliere, seppure il 2016 abbia visto un leggero decremento dovuto quasi certamente al fatto che non tutti gli elettori che hanno espresso la propria preferenza hanno deciso di utilizzare appieno la doppia preferenza di genere. Completamente diverso il comportamento dell'elettorato pentastellato: i tassi di preferenza per i candidati consigliere del Movimento 5 stelle sono e restano incredibilmente bassi.

ТАВ. 13 - Tassi di preferenza nelle elezioni comunali di Bologna, 1999-2016, distinti per area politica.

\begin{tabular}{lccccc}
\hline & Sinistra & Centrosinistra & Centro & Centrodestra & M5S \\
\hline 1999 & 12,8 & 22,1 & & 23,2 & \\
2004 & & 31,3 & & 28,9 & \\
2009 & 29,6 & 23,4 & 28,8 & 7,9 \\
2011 & & 44,4 & 40,7 & 33,1 & 3,3 \\
$2016^{*}$ & 32,1 & 49,0 & & 31,3 & 6,9 \\
\hline
\end{tabular}

Note: * Elezioni con doppia preferenza di genere, il denominatore è quindi moltiplicato per 2.

\section{Che cosa è cambiato e che cosa potrà cambiare}

Le ultime elezioni amministrative segnano per Bologna il definitivo ingresso nell'era della contendibilità (Bartolini 1996). Come abbiamo visto nel paragrafo 2, già se ne era avuta una anticipazione, del tutto eccezionale, nel 1999, con la vittoria nella capitale della "subcultura politica rossa" del candidato civico Giorgio Guazzaloca. Per quanto inattesa, però, quella sconfitta veniva da un candidato peculiare - per storia e profilo (non)politico - e l'egemonia del centrosinistra in città sembrava aver subito una battuta d'attesto momentanea, ma non un definitivo indebolimento. Il principale partito della sinistra, in tutte le sue mutazioni organizzative, continuava a mantenere ben saldo il controllo sull'elettorato bolognese, garantendo stabilità politica e continuità elettorale.

Col passare del tempo, 1'“incantesimo" politico che ha permesso alla filiera PCIPDS-DS-PD di giocare un ruolo dominante, per più di settanta anni, nella realtà pubblica di Bologna sembra essersi definitivamente spezzato. Il voto di appartenenza che ha fatto la fortuna dei partiti di centrosinistra si è andato "assottigliando" nel corso del tempo e oggi ne rimane soltanto una pallida eco. L'abitudine, cioè una sorta di riflesso condizionato, ha preso il posto dell'appartenenza e molti più elettori bolognesi oggi si sentono liberi di cambiare preferenza e partito, spostandosi e saltando tra diverse proposte politi- 
che. Questa tendenza è stata analizzata sotto una duplice prospettiva, quella della domanda elettorale e quella riferita all'offerta partitica.

Sul primo versante, è possibile porre in evidenza alcuni tratti tipici o caratteristici del comportamento elettorale dei bolognesi. Il primo elemento da sottolineare riguarda la maggiore fedeltà dell'elettorato del centrosinistra. Questa compattezza di voto ha rappresentato il vero "asso nella manica" della sinistra bolognese, soprattutto rispetto ai suoi sfidanti del centrodestra, i cui elettori tendono ad essere più mobili $\mathrm{o}$, comunque, meno affidabili da un punto di vista elettorale. Ciò è dovuto anche al ristrutturarsi dell'offerta elettorale che in non pochi casi ha visto i candidati del centrodestra presentarsi in contrapposizione, raccogliendo un discreto numero di consensi, mentre gli elettori della sinistra e del centrosinistra hanno spesso preferito dare il loro voto al candidato della coalizione piuttosto che disperdersi nei mille rivoli rappresentati dai candidati della sinistra più o meno radicale. Ad esempio, per ben due volte il candidato "uscente" del centrodestra, non ricandidato dalla propria coalizione, ha deciso di ripresentarsi da solo: lo ha fatto prima Guazzaloca nel 2009, trascinandosi dietro (e, con ogni probabilità, sottraendo a Cazzola) ben un quarto del proprio elettorato del 2004; lo ha fatto poi Bernardini, trasportando dal 2011 al 2016 circa il 15\% del proprio elettorato.

Il secondo elemento che merita attenzione è, in realtà, una conferma: gli elettori bolognesi sono da sempre, e con pochissime eccezioni, fedeli ai propri partiti di riferimento. Il livello di permeabilità tra i due blocchi principali in competizione (centrodestra e centrosinistra) è basso. Gli interscambi di voti tra i due poli sono piuttosto limitati e, in media, non superano il 4,5\% dell'intero corpo elettorale. Rarissimi sono quindi i "tradimenti", vale a dire i cambi di voto da uno schieramento all'altro, in particolare $i$ passaggi dal centrosinistra al centrodestra. Ciò spiega la bassa competitività elettorale. Ad esempio, tra tutti coloro che nel 2004 avevano sostenuto Cofferati, più dell' $80 \%$ ha confermato la propria preferenza a favore del candidato di centrosinistra. All'opposto, tra gli elettori di Guazzaloca nel 2004 soltanto il $60 \%$ circa scelse Cazzola e la sua coalizione di centrodestra, mentre un quarto dell'elettorato di centrodestra del 2004 decise di seguire Guazzaloca nella sua nuova impresa civica. Ancora, tra coloro che avevano sostenuto Delbono nel 2009, più di 8 elettori su dieci decisero di convergere sulla candidatura di Merola $(85,8 \%)$, confermando sostanzialmente la propria scelta. Solo una piccola parte dell'elettorato di centrosinistra si orientò verso il Movimento 5 Stelle $(9,8 \%)$ e le piccole quote di elettori rimanenti si spostarono verso le liste minori o approdarono nell'astensione. Diverso è il discorso per la coalizione di centrodestra: poco meno di 7 elettori su $10(69 \%)$ che nel 2009 avevano appoggiato Cazzola decisero di votare per il leghista Bernardini, mentre all'incirca l' $11 \%$ sostenne un candidato del polo centrista e il 18,6\% preferì non recarsi alle urne. Questo non significa, però, che il voto dei bolognesi sia totalmente congelato o immobile. Anzi, l'analisi dei flussi elettorali mostra la disponibilità degli elettori a muoversi all'interno di uno stesso schieramento (tra liste più o meno concorrenti), talvolta a preferire opzioni intermedie sfruttando i terzi poli di centro e le liste civiche/indipendenti come $h u b$ per futuri spostamenti oppure, più di recente, a lasciarsi conquistare dalle sirene pentastellate. All'interno di questo scenario, in ottica strategica, diventa cruciale per i partiti bolognesi riuscire a mobilitare innanzitutto il proprio 
elettorato, evitando fughe di voti verso altri attori partitici. L" incantesimo" evocato nel precedente paragrafo sembra essersi spezzato e non è difficile cogliere i segnali di quelli che ne saranno gli effetti in un futuro non troppo lontano.

Il terzo aspetto da evidenziare concerne l'ingresso del Movimento 5 Stelle nel panorama politico locale e poi nazionale. Bologna è la città che ha visto nascere politicamente il Movimento grillino, con il primo Vaffa-day organizzato in Piazza Maggiore nel 2007. Da questo punto di vista, è interessante notare l'evoluzione del Movimento. Il suo exploit nel 2009 era dovuto principalmente alla capacità di intercettare gli elettori scontenti di Cofferati e, in misura minore, di convincere gli astensionisti. Infatti, il $3,7 \%$ di chi aveva votato l'ex segretario della Cgil si era orientato verso Favia. Ciò sta a significare che circa i due terzi dei voti ottenuti da Favia nel 2009 provenivano dagli elettori che in passato avevano sostenuto il candidato del centrosinistra, mentre la quota rimanente dell'elettorato grillino derivava in parte del centrodestra $(9,6 \%)$ e dal mondo degli astensionisti $(19,9 \%)$. Nel giro di appena un biennio, si assiste invece al mutamento del Movimento 5 Stelle, da iniziale "costola della sinistra" a contenitore trasversale degli insoddisfatti provenienti da ogni direzione e partito: all'incirca il $10 \%$ di chi aveva votato Delbono nel 2009, il 7\% tra gli elettori di Guazzaloca e 1'1,5\% tra i sostenitori di Cazzola. Nel frattempo, se da una parte i grillini succhiavano i consensi dei partiti tradizionali, dall'altra parte provavano a fidelizzare i nuovi elettori di recente conquista. Più di due elettori su tre $(73,7 \%)$ tra chi aveva votato il partito di Grillo nel 2009 confermò la propria scelta anche nel 2011, smentendo tutti coloro che lo consideravano un flash party, una scossa passeggera alla politica locale (e nazionale). Seppure con percentuali leggermente più basse, l'elettorato di Bugani gli rimase fedele anche nel passaggio 20112016: il 65,1\% di chi lo aveva votato nel 2011 decise di rivotarlo nel 2016, mentre le restanti quote andarono a disperdersi più o meno equamente tra Borgonzoni, Bernardini $\mathrm{e}$ Martelloni. Dal suo debutto nel 2009 ad oggi, quindi, il "partito di Grillo" ha mostrato sia una grande capacità ricettiva (a Bologna soprattutto nei confronti degli elettori di centrosinistra) sia una notevole abilità nel mantenere i nuovi consensi conquistati all'interno del proprio ambito. Il Movimento 5 Stelle sembra aver individuato un proprio equilibrio organizzativo ed elettorale tale da renderlo un soggetto politico destinato a rimanere sul palcoscenico della politica bolognese (e non solo).

Infine, in quella che per molto tempo è stata considerata la patria del senso civico e del capitale sociale, cioè di una partecipazione diffusa e duratura, è importante interrogarsi sulle caratteristiche dell'astensionismo. Il "partito degli astensionisti" bolognesi, un aggregato elettorale certamente multiforme, negli ultimi venti anni si è allargato, raddoppiandosi (dal 21,1\% nel 1999 al 40,3\% nel 2016). I dati mostrano un calo costante della partecipazione, per tutti i tipi di elezione, con una diminuzione che sfiora i 20 punti percentuali. I risultati della nostra analisi dei flussi mostrano il carattere granitico degli astensionisti bolognesi, i quali non sembrano lasciarsi affascinare dalle offerte dei partiti o dalle tecniche delle campagne elettorali. Mediamente, 1'85\% degli astensionisti conferma la propria (non)scelta, ovvero il proprio non-voto, anche nelle elezioni successive, dimostrandosi completamente insensibile alle trasformazioni dell'offerta partitica. Nelle elezioni comunali la crescita del partito degli astensionisti è legata sostanzialmente a due 
fattori. Da un lato, a fattori contingenti o congiunturali: nel 2009 il 18,5\% degli astensionisti proveniva dall'elettorato scontento del centrosinistra di Cofferati e per il 6,2\% da quello di Guazzaloca; nel 2011 il 13,6\% degli astensionisti era formato da ex-elettori di centrodestra e solo per il 5\% da ex-elettori di sinistra o di centrosinistra; nel 2016, infine, tutte i partiti e le coalizioni in campo, a parte il Movimento 5 Stelle, hanno contribuito all'incirca in egual misura al rafforzamento delle fila dell'astensione (il 20,6\% degli astensionisti proveniva dal centrosinistra, il $23,3 \%$ dal centrodestra, il $28,9 \%$ dai civici Corticelli e Aldrovandi e il $23,7 \%$ da altri candidati). Dall'altro lato, però, si è assistito al rafforzamento della componente strutturale o cronica dell' astensione: nelle ultime cinque tornate elettorali, quasi $1{ }^{\prime} 80 \%$ degli elettori che non si erano recati alle urne nella tornata precedente ha deciso di restare a casa anche alle elezioni successive. Solo una piccola parte del "partito degli astensionisti", quindi, sembra avere un carattere congiunturale, legato alle caratteristiche dei candidati o al tipo di liste in competizione in una determinata tornata elettorale. L'astensionismo strutturale, per di più in crescita negli ultimi decenni, caratterizza il comportamento di quei cittadini che decidono di non decidere, non recandosi alle urne. Come una pianta che si auto-alimenta, anche l'astensionismo degli elettori bolognesi si è rafforzata nel corso degli ultimi venti anni e non mostra alcun segno di cedimento.

Per quanto concerne, invece, il lato dell'offerta partitica, la storia elettorale di Bologna, pur nelle sue storiche peculiarità, riflette le trasformazioni avvenute nel sistema politico italiano nel corso degli ultimi venti anni. Anche a Bologna abbiamo visto affermarsi un bipolarismo frammentato, almeno fino alle elezioni del 2016, così come si era progressivamente formato a livello nazionale. Dopo un cinquantennio in cui, all'indomani del secondo dopoguerra, il sistema partitico si era basato sul predominio elettorale del PCI e sull'assenza dell'alternanza, il nuovo ciclo elettorale inaugurato nel 1995 ha comportato l'avvio di una fase caratterizzata dall'alternanza di governo, non più come possibilità solamente teorica, ma come eventualità empiricamente praticabile. Allo stesso tempo, questa struttura partitica dualista si è accompagnata ad una estrema frammentazione intra-coalizionale, piuttosto accentuata in entrambi i poli, ma più difficile da maneggiare soprattutto per lo schieramento di centrodestra. Sta qui, infatti, la debolezza strutturale del bipolarismo bolognese, vale a dire nel fatto che il polo alternativo al centrosinistra non sempre è riuscito a presentarsi coeso nella competizione elettorale e in grado di rappresentare una vera e propria sfida ai partiti eredi della tradizione comunista.

Ad ogni modo, anche il bipolarismo che si era affermato sotto le Due Torri si è - non sappiamo se e quanto temporaneamente - spezzato all'indomani delle elezioni amministrative del 2016. Con l'ingresso e il consolidamento elettorale del Movimento 5 Stelle, si è aperta una nuova fase multipolare, almeno sul lato della domanda, nel sistema partitico cittadino. Ciò che, però, ha ancora permesso di salvaguardare la sostanza di un confronto bipolare sono stati gli incentivi derivanti da una forma di governo neoparlamentare e dall'elezione diretta del sindaco. La combinazione di questi due fattori, particolarmente distorcenti nel passaggio tra l'arena elettorale e quella governativa nel momento in cui lo scenario partitico si suddivide in tre poli, spiega le diverse conseguenze che il nuovo formato multipolare ha avuto a livello nazionale e locale. Nel primo caso, 
cioè laddove non esistono (o sono deboli) meccanismi elettorali o incentivi istituzionali tendenti al bipolarismo, il confronto tra i partiti è tornato ad assumere i tratti tipici del multipartitismo, per di più frammentato e polarizzato. Nel contesto locale, all'opposto, dove ancora prevale un impianto istituzionale di ispirazione maggioritaria, il tripolarismo in ingresso, cioè nell'arena elettorale e partitica, risulta ancora "forzato" in uno schema bipolare una volta che si entra all'interno delle istituzioni cittadine.

Chiaramente, è ancora troppo presto per prevedere quale dei due scenari finirà per prevalere, ossia se il sistema partitico bolognese finirà per allinearsi perfettamente $\mathrm{a}$ quello nazionale oppure se la forza degli incentivi istituzionali preserverà una struttura bipolare nell'arena esecutiva. Quello che è certo è che il "vecchio" modello politico bolognese, caratterizzato da stabilità politica e prevedibilità elettorale, è ormai definitivamente passato. Anche a Bologna si è aperta la stagione della contendibilità e, forse, sono ormai quasi del tutto scomparsi quei tratti salienti che la rendevano, nel bene e nel male, una città a suo modo eccezionale. 


\section{Riferimenti bibliografici}

Anderlini F. [2006], La città trans-comunista. Appunti di viaggio tra Bologna e altrove, Bologna, Pendragon.

Baldini G., Corbetta P. e Vassallo S. [2000], La sconfitta inattesa. Come e perché la sinistra ha perso a Bologna, Bologna, Il Mulino.

Baldini G. e Legnante G. [2000], Città al voto. I sindaci e le elezioni comunali, Bologna, II Mulino.

Bartolini, S. [1996], «Cosa è "competizione" in politica e come va studiata» in Rivista Italiana di Scienza Politica, 26, 2, pp. 209-267.

Bolgherini S. e Grimaldi, S. (a cura di) [2015], Tripolarismo e destrutturazione. Le elezioni regionali del 2015, Bologna, Istituto Cattaneo.

Bolgherini, S. e Musella, F. [2006], «Le primarie in Italia: ancora e soltanto personalizzazione della politica?», in Quaderni dell'osservatorio elettorale, 55,1, pp. 220-239.

Caciagli M. e Di Virgilio A. (a cura di) [2005], Eleggere il sindaco. La nuova democrazia locale in Italia e in Europa, Torino, UTET.

Campus D. e Pasquino G. [2000], «How to lose a major: the case of Bologna», in Journal of Modern Italian Studies, 5, 1, pp. 22-35.

Cartocci R. [2007], Mappe del tesoro. Atlante del capitale sociale in Italia, Bologna, Il Mulino.

Chiaramonte, A. e Emanuele, V. [2013], «Volatile e tripolare: il nuovo sistema partitico italiano», in L. De Sio, M. Cataldi e F. De Lucia (a cura di), Le elezioni politiche 2013, Roma, Dossier Cise, pp. 95-100.

Chiaramonte, A. e Emanuele, V. [2014], «Bipolarismo addio? Il sistema partitico tra cambiamento e de-istituzionalizzazione», in A. Chiaramonte e L. De Sio (a cura di), Terremoto elettorale, Le elezioni politiche del 2013, Bologna, Il Mulino, pp. 233-262.

Cepernich C. e Vignati R. [2016], «Saper governare non basta: il caso Torino», in M. Valbruzzi e R. Vignati (a cura di), Cambiamento o assestamento? Le elezioni amministrative del 2016, Bologna, Istituto Cattaneo, pp. 23-42.

Galli G. [1967], Il bipartitismo imperfetto. Comunisti e democristiani in Italia, Bologna, Il Mulino.

Galli G. (a cura di) [1968], Il comportamento elettorale in Italia, Bologna, Il Mulino.

Gallagher M. [1991], «Proportionality, Disproportionality and Electoral Systems», in Electoral Studies, 10, 1, pp. 33-51.

Goodman L. A. [1953], «Ecological Regressions and Behavior of Individuals», in American Sociological Review, 18, 6, pp. 663-664.

Gramsci A. [1975], Quaderni dal carcere, Torino, Einaudi.

Grandi R. e Vaccari C. [2004], Cofferati anch'io, Un anno di campagna elettorale a Bologna, Milano, Baldini Castoldi.

Laakso M. e Taagepera R. [1979], «“Effective” number of parties. A Measure with Appli- 
cation to West Europe», in Comparative Political Studies, 12, 1, pp. 3-27.

Natalizia G. e Vignati R. [2016], «Roma: una svolta annunciata», in M. Valbruzzi e R. Vignati (a cura di), Cambiamento o assestamento? Le elezioni amministrative del 2016, Bologna, Istituto Cattaneo, pp. 151-170.

Pasquino G. [2011], Quasi sindaco. Politica e società a Bologna 2008-2010, Reggio Emilia, Diabasis.

Pasquino G. e Valbruzzi M. [2015], A Changing Republic. Politics and Democracy in Italy, Novi Ligure, Edizioni Epoké.

Putnam R. D. et al.[1993], La tradizione civica nelle regioni italiane, Milano, Mondadori.

Ramella S. [2005], Cuore rosso? Viaggio politico nell'Italia di mezzo, Roma, Donzelli.

Sandri G. e Valbruzzi M. [2012], Le primarie di Bologna: caso di studio, di scuola o di successo?, paper presentato al XXVI Convegno della Società Italiana di Scienza Politica, Università Roma Tre, 13 - 15 settembre.

Sani G. [1992], «La destrutturazione del mercato elettorale», in Rivista Italiana di Scienza Politica, 22, 3, pp. 539-565.

Sartori G. [1976], Parties and Party Systems. A framework for analysis, Cambridge, Cambridge University Press.

Schadee H.m. A. e Corbetta P. [1984], Metodi e modelli di analisi dei dati elettorali, Il Mulino, Bologna.

Seddone A. e Valbruzzi M. [2010], «Le elezioni primarie tra partiti e partecipazione: analisi comparata dei casi di Bologna e Firenze», Polis, 24, 2, pp. 195-224.

Tronconi, F. [2015], «Bye-Bye Bipolarism: The 2015 Regional Elections and the New Shape of Regional Party Systems in Italy», South European Society and Politics, 20, 4, pp. 553-571.

Vassallo S. [2013], «Ricchezza, civismo, forza dei governi: il divario in cerca di una spiegazione», in S. Vassallo (a cura di), Il divario incolmabile. Rappresentanza politica e rendimento istituzionale nelle regioni italiane, Bologna, Il Mulino, pp. 9-34. 